\title{
Photonic RF and microwave spectral filters based on transversal filters with Kerr microcombs
}

David Moss ( $\nabla$ dmoss@swin.edu.au )

Swinburne University of Technology

\section{Research Article}

Keywords: Microwave photonics, micro-ring resonators, spectral filters

Posted Date: June 4th, 2021

DOl: https://doi.org/10.21203/rs.3.rs-582860/v1

License: (c) (i) This work is licensed under a Creative Commons Attribution 4.0 International License. Read Full License 


\section{Abstract}

Integrated Kerr microcombs are emerging as a powerful tool as sources of multiple wavelength channels for photonic RF and microwave signal processing mainly in the context of transversal filters. They offer a compact device footprint, very high versatility, large numbers of wavelengths, and wide Nyquist bands. Here, we present our recent progress on Kerr microcomb-based photonic RF and microwave reconfigurable filters, based both on transversal filter methods and on RF to optical bandwidth scaling. We compare and contrast results achieved with wide comb spacing combs $(200 \mathrm{GHz})$ with more finely spaced $(49 \mathrm{GHz})$ microcombs. The strong potential of optical micro-combs for RF photonics applications in terms of functions and integrability is also discussed.

\section{Introduction}

Nonlinear optics for all-optical signal processing has proven to be an extremely powerful approach, particularly when implemented in photonic integrated circuits based on highly nonlinear materials such as silicon [1, 2]. All optical signal processing functions include all-optical logic [3], demultiplexing from $160 \mathrm{~Gb} / \mathrm{s}$ [4] to over $1 \mathrm{~Tb} / \mathrm{s}$ [5], optical performance monitoring via slow light [6-7], all-optical regeneration $[8,9]$, and others [10-16]. CMOS compatible platforms are centrosymmetric, and so nonlinear devices have been based on third order nonlinear processes including third harmonic generation [11,17-21] and, the Kerr nonlinearity $\left(n_{2}\right)[1,2]$. The efficiency of Kerr based all-optical devices depends on the waveguide nonlinear parameter, $\gamma=w n_{2} / c A_{\text {eff }}$ where $\mathrm{n}_{2}$ is the Kerr nonlinearity and $\mathrm{A}_{\text {eff }}$ is the waveguide effective area). Although silicon can achieve extremely high values of $\gamma$, it suffers from high nonlinear losses due to two-photon absorption (TPA) and the resulting free carriers [2]. Even if the free carriers are eliminated by $p-i-n$ junctions, silicon's poor intrinsic nonlinear figure of merit (FOM $=n_{2} /(\beta \lambda)$, where $\beta$ is the TPA) of around 0.3 in the telecom band is far too low to achieve high performance. While TPA can be turned to advantage for some all-optical functions [22-24], for the most part silicon's low FOM in the telecom band is a limitation. This has motivated research on a range of alternate nonlinear platforms highlighted by perhaps by the chalcogenide glasses [25-35]. While offering advantages, these platforms are not compatible with the silicon computer chip industry - CMOS (complementary metal oxide semiconductor).

In 2008 new CMOS compatible platforms for nonlinear optics, including silicon nitride $[35,36]$ and Hydex [37-47] were introduced with negligible nonlinear absorption in the telecom band, a moderate nonlinear parameter and extremely high nonlinear figure of merit, which are ideal for micro-comb generation [47]. Following the first report of Kerr frequency comb sources in 2007 [48], the first integrated CMOS compatible integrated optical parametric oscillators were reported in 2010 [36, 37], and since then this field has exploded [47]. Many cutting-edge applications have been demonstrated based on CMOScompatible micro-combs, ranging from filter-driven mode-locked lasers [49-52] to quantum physics [5358]. Most recently, Kerr micro-combs have demonstrated their enormous potential for sources for ultrahigh bandwidth coherent optical fiber communications [59], optical frequency synthesis [60] and many other applications [61-68]. The success of these new CMOS platforms as well as other CMOS 
platforms such as amorphous silicon [69] and silicon rich silicon nitride (SRSN) [70] arises from a combination of their low linear loss, moderate to high nonlinearity and low or even negligible nonlinear loss (TPA).

Photonic RF techniques are an important application of all-optical signal processing. They have attracted great interest during the past two decades since they offer ultra-high RF bandwidths, low transmission loss and strong immunity to electromagnetic interference. They have found wide applications ranging from radar systems to communications [71-100]. There are a very wide variety of approaches to realizing photonic RF filters, including ones that map the optical filter response onto the RF domain [80-95], highlighted by on-chip (waveguide based) stimulated Brillouin scattering [83-88]. This approach has achieved extremely high performance in terms of RF resolution - as high as $32 \mathrm{MHz}$ - and a stopband rejection $>55 \mathrm{~dB}$. Another key approach focuses on reconfigurable transfer functions for adaptive signal processing based on transversal filters [86-91]. These operate by generating weighted and progressively delayed replicas of the RF signal in the optical domain and then combining them upon photo-detection. Transverse filters are capable of achieving arbitrary RF transfer functions by simply changing the tap weights, and so are attractive for the implementation of advanced adaptive and dynamic RF filters. Typically, discrete laser arrays [92-94] or Bragg grating arrays [95,96] have been employed to supply the needed taps. However, while offering many advantages, these approaches result in significantly increased complexity as well as reduced performance due to the limited number of available taps. Alternative approaches, including those based on optical frequency comb sources achieved by electrooptic (EO) modulation, can help mitigate this problem, but they require cascaded high frequency EO [9799] or Fabry-Perot EO [100] modulators that in turn require high-frequency RF sources. Of these approaches, EO comb-based methods have been in use the longest and have demonstrated many powerful functions.

Integrated optical Kerr frequency comb sources, or "microcombs" [101-125] have come into focus as a fundamentally new and powerful tool due to their ability to provide highly coherent multiple wavelength channels with a very high degree of wavelength spacing control, from a single source. They originate via optical parametric oscillation in monolithic micro-ring resonators (MRRs), offer significant advantages over more traditional multi-wavelength sources for RF applications. They can greatly increase the capacity of communications systems and allow the processing of RF spectra for very wide range of advanced signal processing functions [125-144]. They have the advantage of being able to generate frequency combs with much higher spacings than electro-optic combs. In fact, EO combs and microcombs are in many ways complementary - the former focusing on finer wavelength spacings from 10 's of $\mathrm{MHz}$ to $10-20 \mathrm{GHz}$, while the microcombs typically have much wider spacings from $10-20 \mathrm{GHz}$ up to 100 's of $\mathrm{GHz}$ and even terahertz spacing. Wider comb spacings yield much wider Nyquist zones for larger RF bandwidths, whereas finer spacings potentially can yield much larger numbers of wavelengths or RF "taps", at the expense of much more limited Nyquist zones and hence RF bandwidths. Microcombs have the potential to provide a much higher number of wavelengths, an ultra-large FSR, as well as greatly reduced footprint and complexity. In particular, for RF transversal functions, the number of wavelengths dictates the available channel number of RF time delays, and thus with microcombs, the 
performance of RF filters and other systems such as beamforming devices can be greatly enhanced in terms of the quality factor and angular resolution, respectively. In addition, other approaches to filtering such as RF bandwidth scaling [138] have a given bandwidth for each wavelength channel, and so the total operation bandwidth (i.e., the maximum bandwidth of the input RF signal that can be processed) will depend on the number of wavelengths, and hence will be greatly enhanced with microcombs. Based on these advantages, a wide range of RF applications have been demonstrated, such as optical true time delays [130], transversal filters [134, 138], signal processors [127,135], and channelizers [137].

Here, we report RF and microwave spectral filters based on transversal filtering methods that exploit integrated Kerr microcomb devices. We demonstrate fixed and tunable bandpass filters as well as less common filters such as gain equalizing, low pass etc.. We discuss the tradeoffs between using very widely spaced microcombs at $200 \mathrm{GHz}$ with recently reported $[127,130,134,138]$ record low FSR spaced microcombs at $49 \mathrm{GHz}$, based on soliton crystals. We report new advances of RF and microwave filtering functions made possible through the use of microcombs, highlighting their potential and future possibilities, contrasting the different methods and use of the differently spaced microcombs. The RF transversal filters based on $200 \mathrm{GHz}$ Kerr micro-combs achieved a high degree of versatility and dynamic reconfigurability. However, their relatively large comb spacing arising from the large free spectral range (FSR) of $\sim 1.6 \mathrm{~nm}(\sim 200 \mathrm{GHz})$ restricts the number of taps to less than 21 within the 30nm wide bandwidth of the $\mathrm{C}$-band. This is an important consideration since this approach requires optical amplifiers and optical spectral shapers which are more readily available for wavelengths in the C-band. The limitation in the number of taps has limited the performance of micro-comb based transversal RF filters in terms of frequency selectivity, tuning resolution, dynamic versatility (filter shapes), as well as the performance of RF photonic signal processors. In order to improve on this, we demonstrated micro-combbased photonic RF transversal filters with record high numbers of taps, featuring up to 80 wavelengths over the C-band $[127,130,134,138]$. This is the highest number of wavelengths so far reported for microcomb-based RF transversal filters, enabled by a record low $49 \mathrm{GHz}$-free-spectral-range integrated Kerr micro-comb source. This resulted in a $Q_{\mathrm{RF}}$ factor for the RF bandpass filter of four times higher than the $200 \mathrm{GHz}$ FSR results [134]. Further, by programming and shaping the Kerr optical micro-comb, we achieve [134] RF filters with a high out-of-band rejection of up to $48.9 \mathrm{~dB}$ using Gaussian apodization, as well as a significantly improved tunable centre frequency covering the RF spectra range (from $0.05 \times F S R_{\mathrm{RF}}$ to $\left.0.40 \times F S R_{\mathrm{RF}}\right)$. We also demonstrate an adaptive photonic RF filter with highly reconfigurable 3-dB bandwidths (from 0.5 to $4.6 \mathrm{GHz}$ ) and arbitrary filter shapes. Our experimental results agree well with theory, verifying the feasibility of our approach towards the realization of high performance advanced adaptive RF transversal filters with potentially reduced cost, footprint, and complexity than other solutions.

\section{Integrated Kerr Microcombs}

The generation of microcombs is a complex process that generally relies on high nonlinear optical parameters, low linear and nonlinear loss as well as engineered anomalous dispersion. Diverse platforms 
have been developed for microcomb generation [108] such as silica, magnesium fluoride, silicon nitride, and doped silica glass. The MRRs used in the experiments presented here were fabricated using CMOS compatible fabrication processes, with Q factors of over 1.2 million and radii of $\sim 592 \mu \mathrm{m}$ and $\sim 135 \mu \mathrm{m}$, corresponding to FSRs of $\sim 0.4 \mathrm{~nm}(\sim 49 \mathrm{GHz})$ and $\sim 1.6 \mathrm{~nm}(\sim 200 \mathrm{GHz})$, respectively $[130,139]$.

The MRRs used to generate the Kerr optical micro-combs are shown in Figure 1a for the 200GHz FSR combs and $1 \mathrm{~b}$ for the $49 \mathrm{GHz}$ combs. They were both fabricated on a high-index doped silica glass platform using CMOS-compatible fabrication processes [104, 105]. First, high-index ( $n=\sim 1.7$ at 1550 $\mathrm{nm}$ ) doped silica glass films were deposited using plasma enhanced chemical vapour deposition, then patterned by deep ultraviolet photolithography and etched via reactive ion etching to form waveguides with exceptionally low surface roughness. Finally, silica $(\mathrm{n}=\sim 1.44$ at $1550 \mathrm{~nm})$ was deposited as an upper cladding. The device architecture typically uses a vertical coupling scheme where the gap (approximately $200 \mathrm{~nm}$ ) can be controlled via film growth - a more accurate approach than lithographic techniques. The advantages of our platform for optical micro-comb generation include ultra-low linear loss $\left(\sim 0.06 \mathrm{~dB} \llbracket \mathrm{cm}^{-1}\right)$, a moderate nonlinear parameter $\left(\sim 233 \mathrm{~W}^{-1} \triangle \mathrm{km}^{-1}\right)$, and in particular a negligible nonlinear loss up to extremely high intensities $\left(\sim 25 \mathrm{GW} \Downarrow \mathrm{cm}^{-2}\right)$. Due to the ultra-low loss of our platform, the MRR features narrow resonance linewidths, corresponding to a quality factor of $\sim 1.5$ million (Fig. 1) for both MRRs. After packaging the device with fiber pigtails, the through-port insertion loss was as low as $0.5 \mathrm{~dB} /$ facet, assisted by on-chip mode converters. The radius of the $49 \mathrm{GHz}(0.4 \mathrm{~nm}) \mathrm{FSR}$ MRR was $\sim 592 \mu \mathrm{m}$, while the $200 \mathrm{GHz}$ FSR device had a radius of $135 \mu \mathrm{m}$. The $200 \mathrm{GHz}$ FSR device was the basis of our early work from the first report in 2008 on nonlinear optics in MRRs [115] followed by our work on micro-combs $[104,105]$. In particular, the reports of microcombs in $2010[103,104]$ were the first reports of optical microcombs based on a fully integrated photonic chip platform, following the first report of microcombs in toroidal resonators [101]. Our work on true time delays [130] reported the first integrated microcomb able to generate soliton states, with an FSR $<100 \mathrm{GHz}$. The record small optical free spectral range of the $49 \mathrm{GHz}$ FSR MRR greatly expanded the number of wavelengths (channels) available over the telecommunications band to as many as 80 wavelengths, or taps for microwave applications - much larger than previous reports.

To generate microcombs with the $200 \mathrm{GHz}$ device, the CW pump power is typically amplified and then the wavelength is swept from blue to red. When the detuning between the pump wavelength and MRR's cold resonance wavelength becomes small enough such that the intracavity power reaches a threshold, a modulation instability driven oscillation is initiated [108]. As the detuning is changed further, single-FSR spaced microcombs are generated. Achieving rigorous single soliton states in micro-combs via dissipative Kerr solitons (DKS), while well understood [107], is nonetheless very challenging. It requires both frequency and amplitude sweeping in both directions in order to "capture" the soliton states out of the initially chaotic states. Our initial work on microcomb applications were based on the $200 \mathrm{GHz}$ microcombs operating in a partially coherent state that, while not exhibiting rigorous soliton behaviour, was nonetheless a relatively low noise state that avoided the chaotic regime [108] and was more than adequate for microwave applications. The spectrum shown in Figure 1 is typical of the $200 \mathrm{GHz}$ spectra 
that we employed for our initial microwave work, and clearly shows that it is not a rigorous soliton state. Our work on the $49 \mathrm{GHz}$ FSR combs is based on soliton crystals $[109,130,134,138]$ resulting from mode crossings in the $\mathrm{C}$-band. Soliton crystals are in fact much easier and robust to generate than DKS states and indeed even our partially coherent $200 \mathrm{GHz}$ states. They are achievable through simple manual tuning of the pump laser wavelength which is impossible with any other state. One reason for this is that the internal cavity optical energy of the soliton crystal state is very close to that of the chaotic state, and so when transitioning from one to the other, there is a very small shift in internal energy and hence resonant wavelength jump. It is this "jump" in both internal energy and resonant wavelengths of the microresonator that primarily is responsible for the challenge in realizing DKS states. The efficiency of the soliton crystal states - ie., the energy in the comb lines relative to the pump wavelength - is also much higher than the DKS states (the single soliton states in particular). The tradeoff is that the output spectrum of soliton crystals is not flat but exhibits a characteristic "curtain-like" pattern. While this typically requires a spectral flattening step, it has not posed a fundamental barrier to achieving a wide range of high performance RF and microwave processing functions. Soliton crystals also result from mode-crossings in the resonator, which can be challenging to design, rather than simply requiring the anomalous dispersion that DKS states need.

To generate soliton crystal Kerr micro-combs, we adjusted the polarization and wavelength of the pump light to one of the TE resonances of the MRR typically the resonance at 1553.2 nm [130,134] with the pump power set at $\sim 30.5 \mathrm{dBm}$. When the detuning between the pump wavelength and the cold resonance became small enough, such that the intra-cavity power reached a threshold value, modulation instability (MI) driven oscillation was initiated. Primary combs were thus generated with the spacing determined by the MI gain peak - mainly a function of the intra-cavity power and dispersion. As the detuning was changed further, distinctive 'fingerprint' optical spectra were observed (Fig. 2). These spectra are similar to what has been reported from spectral interference between tightly packed solitons in the cavity - so called "soliton crystals" [104, 105]. An abrupt step in the measured intracavity power (Fig. 3(b)) was observed at the point where these spectra appeared, as well as a dramatic reduction in the RF intensity noise (Fig. 3(c)). Together with the spectra shapes, we take these observations as indicative of soliton crystal formation [105], although to conclusively demonstrate this one would need to perform time resolved pulse autocorrelation measurements. The key issue for our experiments was not the specific nature of the micro-comb state of oscillation so much as the low RF noise and high coherence, which were relatively straightforward to achieve through adiabatic pump wavelength sweeping. We found that it was not necessary to achieve any specific state, including either soliton crystals or single soliton states in order to obtain high performance - only that the chaotic regime [108] should be avoided. Nonetheless the soliton crystal states provided the lowest noise states of all of our microcombs, and was used as the basis for a microwave oscillator with low phase-noise [128]. This is important since there are a much wider range of coherent low RF noise states that are more readily accessible than any specific soliton related state [108].

\section{Rf Transversal Filter Theory}


The transversal structure, similar to the concept of finite impulse response digital filters, is a fundamental tool for photonic RF signal processing. With the proper design of the tap weights, any transfer function can be arbitrarily realized for different signal processing functions such as bandpass filters, differentiators and Hilbert transformers [139]. The transfer function of the microwave photonic filter (MPF) can be described as (Figure 3): See formula 1 in the supplementary files.

where $\omega$ is the angular frequency of the input RF signal, $N$ is the number of taps, $a_{n}$ is the tap coefficient of the $n_{t h}$ tap, and $T$ is the time delay between adjacent channels (see Fig. 3). The Nyquist frequency of the MPF is given by $f_{N y q u i s t}=1 / 2 T$. By imposing calculated tap coefficients $a_{n}$ on each wavelength channel, reconfigurable MPFs with arbitrary spectral transfer functions can be implemented. Figure 3 shows a schematic diagram of the MPFs based on wavelength division multiplexing. The large number of wavelengths offered by the microcombs provides significant advantages in the performance of transversal signal processors. The progressively delayed RF replicas are termed "taps", and the number of taps directly determines the resolution, or the $Q_{\mathrm{RF}}$ factor, of the system. By properly setting the tap coefficients, a reconfigurable transversal filter with diverse transfer functions can be achieved, including Hilbert transformers [127, 136], differentiators [135], and bandpass filters [132, 134, 139].

As Fig. 3 shows, the core of the microcomb-based transversal structure mainly contains two operations: broadcast and then delay. The input RF signal is first broadcast onto equally spaced wavelength channels via optoelectronic modulation to generate multiple replicas of the RF input. The replicas are then transmitted through a dispersive medium to acquire wavelength-sensitive delays. Second-order dispersion, which yields a linear relationship between the wavelength and the delay, is generally employed to progressively separate the replicas. The delayed signals can be either individually converted into electronic signals or summed upon photodetection. The former can be implemented through wavelength demultiplexers and photodetector arrays to perform the function of RF true time delays [130], while the latter can serve as transversal filters for RF signal processing [139].

Figure 4 shows the experimental setup for a system based on an integrated Kerr optical comb source with a $200 \mathrm{GHz}$ FSR (Figure 4a) and 49GHz FSR (Figure 4b). The main difference between the two experimental setups is the use of a second waveshaper for the $49 \mathrm{GHz}$ FSR comb, used to pre-flatten the scallop-shaped comb spectrum that is a distinctive hallmark of soliton crystal combs. The micro-comb, in both cases generated by the on-chip MRR, is then amplified and fed to either a single $(200 \mathrm{GHz}$ comb) or dual $(49 \mathrm{GHz}$ comb) waveshapers for channel equalization and weighting. The power of each comb line is manipulated by the waveshapers to achieve appropriately weighted tap coefficients. To increase the accuracy, we adopted a real-time feedback control path to read and shape the power of the comb lines accurately. The comb line powers were first detected by an optical spectrum analyser and then compared with the ideal tap weights. This allowed us to generate an error signal that was fed back into the waveshaper to calibrate the system and achieve accurate comb shaping. The processed comb lines were then divided into two parts according to the algebraic sign of the tap coefficients, and then fed into a $2 \times 2$ balanced MZM biased at quadrature. The $2 \times 2$ balanced MZM could simultaneously modulate the input 
RF signal on both positive and negative slopes, thus yielding replicas of the input RF signal with phase and tap coefficients having either algebraic sign.

For the $200 \mathrm{GHz}$ comb based system, the modulated signal produced by the MZM then went through $\sim 2.122-\mathrm{km}$ of standard SMF, where the dispersion was $\sim 17.4 \mathrm{ps} /(\mathrm{nm} \nabla \mathrm{km})$, corresponding to a minimum time delay $T$ of $\sim 59$ ps between adjacent taps (with the channel spacing of the time delay lines set equal to the FSR of the MRR), yielding a Nyquist frequency of $\sim 8.45 \mathrm{GHz}$ for the MPF. We note that the operational bandwidth of the MPF was determined by the Nyquist frequency, which could be easily enlarged by decreasing the time delay and, owing to the large FSR of the compact MRR, could potentially reach over $\sim 100 \mathrm{GHz}$. Finally, the weighted and delayed taps were combined upon detection and converted back into RF signals at the output.

For the $49 \mathrm{GHz}$ comb based filter, the signal went through $\sim 5-\mathrm{km}$ of standard single mode fibre (SMF) to provide the progressive tap delays. The dispersion of the fibre was the same $(17 \mathrm{ps} / \mathrm{nm} / \mathrm{km})$, yielding a time delay $T$ of $\sim 34.8$ ps between adjacent taps, yielding an operation bandwidth (i.e., the Nyquist frequency, half of $F S R_{\mathrm{RF}}$ ) of $\sim 14.36 \mathrm{GHz}$ for the transversal filter. This operation bandwidth can be easily enlarged by decreasing the time delay (e.g., using a shorter spool of SMF), at the expense of a reduced tuning resolution. However, the maximum operational bandwidth of the transversal filter is limited by the comb spacing. Significant crosstalk between adjacent wavelength channels (or taps) occurs for RF operation beyond $24.5 \mathrm{GHz}$ - half of the microcomb's spacing $49 \mathrm{GHz}$. This issue can be addressed by employing a microcomb source with a larger comb spacing, although at the expense of providing fewer comb lines/taps across the C-band. Finally, as for the $200 \mathrm{GHz}$ based system, the weighted and delayed taps were combined and converted back into RF domain via a high-speed photodetector (Finisar, $40 \mathrm{GHz}$ bandwidth).

\section{Fixed Filters}

Our initial work on micro-comb based MPFs was based on a $200 \mathrm{GHz}$ microcomb. We achieved noticeably better performance of some parameters such as the quality factor $\left(Q_{R F}\right)$, of the bandpass filter (the ratio of its 3-dB bandwidth to $T$ ) as a result of the large number of taps provided by the optical comb source. In order to establish a benchmark for the bandpass filter, we used uniform tap coefficients. Figure 5 shows a simulated all-ones MPF with different numbers taps for the $200 \mathrm{GHz}$ FSR comb, and as can be seen, the 3$\mathrm{dB}$ bandwidth decreases greatly as the tap number increases. The shaped optical combs exhibit a good match between experiment (red solid line) and theory (green crossing). The RF response of the all-ones MPF was characterized by a vector network analyser (VNA, Anritsu 37369A) (Fig. 5) confirming the increase in $Q_{R F}$ factor when expanding the tap number from 4 to 20 .

By comparison, the $49 \mathrm{GHz}$ FSR combs yielded far superior performance in frequency selectivity, or resolution of the transversal filters, brought about by the large number of taps and represented by the $Q_{\mathrm{RF}}$ factor. Figure 6 shows the micro-comb wavelengths used to implement low-pass sinc filters featuring equal tap weights (i.e., $\left.h_{\operatorname{sinc}}(n)=1\right)$ for different numbers of taps. We measured the 3 -dB bandwidth 
( $\left.B W_{\text {sinc }}\right)$ to calculate the corresponding $Q_{\mathrm{RF}}$ factor $\left(Q_{\mathrm{RF}}=B W_{\text {sinc }} / F S R_{\mathrm{RF}}\right)$. In the experiments, we selected different numbers of taps ranging from 2 to 80 . The corresponding optical spectra are shown in Fig. 6. The RF transmission spectra of the sinc filter (Fig. 6(a)) were measured by a vector network analyser (VNA, Anritsu 37369A), and showed good agreement with theory (Fig. 6(b)). The measured $B W_{\text {sinc }}$ decreased from 3.962 to $0.236 \mathrm{GHz}$ when the tap number was increased from 2 to 80 , indicating a greatly enhanced $Q_{\mathrm{RF}}$ of up to 73.7 with 80 taps - four times larger than the $200 \mathrm{GHz}$ results.

Figure 7 shows the dependence of the RF filter Q factor and bandwidth on the number of taps, contrasting the results obtained with the $200 \mathrm{GHz}$ device versus the $49 \mathrm{GHz}$ device. In advancing from 20 taps for the $200 \mathrm{GHz}$ device to 80 taps for the $49 \mathrm{GHz}$ device, the $\mathrm{Q}$ factor is improved from around 20 to almost 80 , with a corresponding reduction in bandwidth from about $900 \mathrm{MHz}$ to under $200 \mathrm{MHz}$. Perhaps just as important is the out-of-band crosstalk. For the $200 \mathrm{GHz}$ device this is comparatively quite high at $-10 \mathrm{~dB}$ - probably too high for most practical applications. However, for the $49 \mathrm{GHz}$ device this drops to below $-25 \mathrm{~dB}$ which is a very significant improvement, rendering this device appropriate for practical applications. The theoretical $B W_{\text {sinc }}$ and $Q_{\mathrm{RF}}$ as a function of the tap number are shown in Fig 7 as well as the experimental $Q_{R F}$ factor and 3-dB bandwidth for the $200 \mathrm{GHz}$ and $49 \mathrm{GHz}$ devices. As can be seen, $Q_{\mathrm{RF}}$ increases linearly with the tap number and the 3-dB bandwidth decreases greatly as the tap number increases, further confirming the significant improvement of the frequency selectivity or resolution (reflected by $Q_{\mathrm{RF}}$ ) brought about by the large tap number used here. The shaped optical combs (Fig. 6) also exhibit a good match between experiment (red solid line) and theory (green crossing), indicating that the comb lines were accurately shaped.

Finally, we note that it is not always a case of a smaller FSR comb being better. The $200 \mathrm{GHz}$ devices are capable of reaching RF bandwidths, particularly in tunability, approaching $100 \mathrm{GHz}$, or the Nyquist zone, whereas the $49 \mathrm{GHz}$ devices are limited to about $25 \mathrm{GHz}$. We discuss tunable RF filters in the next section.

\section{Tunable Filters}

Tunable bandpass filters are an important application in the RF signal processing field. While in conventional schemes this is generally obtained by physically varying the physical time delay, here we achieve this by only varying the tap coefficients. This illustrates the versatility and reach of micro-comb transversal filters, greatly reducing the complexity and instability of the system.

We begin by focusing on the $200 \mathrm{GHz}$ FSR based filters, although the approach is the same for the $49 \mathrm{GHz}$ device. We employ the Remez algorithm [144] to determine the tap coefficients for the bandpass MPF with different center frequencies. Theoretically, the center frequency $\left(f_{c}\right)$ and transition bandwidth $\left(B W_{t r}\right)$ are critical to determining the $Q_{R F}$ factor and the required taps, and so we divide the design of the tunable MPF into two steps. First, the required dynamic range of the generated comb (i. e., the difference between the maximum and minimum power of the comb lines) and the necessary number of taps are calculated as functions of $f_{c}$ and $B W_{t r}$. We account for practical limitations such as a dynamic range of $<20 \mathrm{~dB}$ and 
a tap number $<20$ (for the $200 \mathrm{GHz}$ based combs) in order to determine the available sets of $f_{c}$ and $B W_{t r}$ Secondly, we calculated the bandwidth and $Q_{R F}$ factor such that the optimized sets of $f_{c}$ and $B W_{t r}$ were subject to these practical restrictions. As a result, we are able to achieve a tunable MPF with optimized performance by just re-programming the tap weights, without the need for physical tunable delay lines. Thus, the advantages enabled by the use of Kerr combs are clear. In turn, this allowed a large number of wavelengths, providing a large number of taps that resulted in improved $Q_{R F}$ factors of the MPFs. For the $200 \mathrm{GHz}$ based device, we select 5 sets of tap coefficients resulting in 5 different center frequencies for the tunable MPF. Figure 8 verifies theoretically that the designed tunable MPFs could achieve acceptable performance. Figure 8 shows that the filter is able to achieve a tuning range from $2 \mathrm{GHz}$ to $6 \mathrm{GHz}$ with a bandwidth of about $1 \mathrm{GHz}$. Figure 8 also shows the corresponding results for the $49 \mathrm{GHz}$ device, showing a dynamic tuning range from $2 \mathrm{GHz}$ to about $14 \mathrm{GHz}$ and with a bandwidth of $533 \mathrm{MHz}$.

\section{Versatile And High Performance Filters}

The large number of wavelengths provided by the $49 \mathrm{GHz}$ comb opens up the possibility of realizing both very high performance filters, in terms of resolution and out-of-band crosstalk, as well as versatility for providing almost arbitrary filter shapes. Figure 9 shows the filter weights and resulting filter spectral profile of a gaussian apodized bandpass filter using 80 taps. To improve the performance of the transversal filter in terms of out-of-band rejection, a Gaussian apodization was applied to the sinc filter [134], which was achieved by modifying the original discrete impulse response (i.e., tap weights) to the product of itself and a Gaussian function, given by see formula 2 in the supplementary files section.

where $\sigma$ is the root mean square width of the Gaussian function. Figure 9 shows the shaped comb spectra and the corresponding RF transmission spectra. As a function of decreasing $\sigma$, the main-tosecondary sidelobe ratio (MSSR) of the sinc filter increased from 26.4 to $48.9 \mathrm{~dB}$, although at the expense of a deteriorated $Q_{\mathrm{RF}}$ factor. While illustrating the capability of our transversal filter to achieve high out-ofband rejection, it also indicates that a large number of taps would be needed if both a very high $Q_{\mathrm{RF}}$ factor and MSSR are simultaneously required. With the ability to offer a large number of taps potentially over 200 in the $C+L$ bands, micro-combs serve as a highly attractive approach to meet these demands. We note that the experimental results for $\sigma=10$ were limited by our measurement noise floor of the VNA, and that up to $74.5 \mathrm{~dB}$ can in principle be achieved. Finally, Figure 10 shows RF filters for variable transmission across the passband (for gain equalizing functions etc) as well as a variable bandpass filter with the passband varying from $500 \mathrm{MHz}$ to over $4.6 \mathrm{GHz}$. All of these filtering functions were achieved solely by varying the wavelength tap weights, with no changes in any of the physical parameters otherwise.

The results reported here highlight the advantages of using Kerr micro-combs as the basis for RF photonic microwave transversal filters. It also illustrates the tradeoffs between using widely spaced microcombs $(200 \mathrm{GHz})$ and record low spaced microcombs $(49 \mathrm{GHz})$ in terms of performance results. The greater number of lines supplied by the $49 \mathrm{GHz}$ comb ( 80 versus 20 for the $200 \mathrm{GHz}$ device) yield 
significantly better filter performance in terms of resolution, or Q-factor, tunability and cross-talk. On the other hand the $49 \mathrm{GHz}$ device is more limited in operational bandwidth, being restricted to roughly the Nyquist zone of $25 \mathrm{GHz}$. The $200 \mathrm{GHz}$ device is able to reach RF frequencies that are well beyond what conventional electronic microwave technology can achieve.

\section{Conclusion}

Microcombs have shown their powerful capabilities for RF signal processing due to their large number of comb lines and compact footprint. We believe that they will bring further benefits to RF photonics, particularly in two aspects. First, the coherent nature of the soliton states will enable more advanced RF functions such as wideband frequency conversion and clock generation. Secondly, globally established CMOS fabrication platforms, which can perform hybrid integration of the microcomb source and $\mathbb{\nabla}-\mathbb{Z}$ devices, will potentially enable monolithic integration of the entire RF system. Soliton crystals have achieved success for a wide range of applications including microwave and quantum photonics as well as incorporating 2D material [145-235].

We demonstrate record performance and versatility for microcomb-based photonic RF transversal filters by employing $200 \mathrm{GHz}$ and $49 \mathrm{GHz}-\mathrm{FSR}$ integrated optical micro-comb sources capable of providing record high numbers of wavelengths, or taps - up to 80 over the C-band- as well as a very high comb wavelength spacings for ultrahigh RF frequency applications. We achieve $Q_{\mathrm{RF}}$ factors that are four times larger for the $49 \mathrm{GHz}$ devices compared with filters based on the $200 \mathrm{GHz}$ combs, as well as a high out-ofband rejection of up to $48.9 \mathrm{~dB}$ using Gaussian apodization. We also report RF filters with tunable centre frequencies achieved by only varying the tap weights. We cover the RF spectral range (from $0.05 \times F S R_{\mathrm{RF}}$ to $0.40 \times F S R_{\mathrm{RF}}$, or 1.4 to $11.5 \mathrm{GHz}$ ), as well as tunable $3 \mathrm{~dB}$ bandwidths ranging from 0.5 to $4.6 \mathrm{GHz}$. Finally, we demonstrate highly reconfigurable filter shapes with positive and negative slopes across the passband as well as rectangular bandpass filter shapes. The experimental results agree well with theory, verifying that our transversal filter is a competitive approach towards achieving advanced adaptive RF transversal filters with broad operational bandwidths, high frequency selectivity, high reconfigurability, and potentially reduced cost and footprint, all of which are critical issues for modern radar and communications systems.

\section{Declaration}

Competing interests: The authors declare no competing interests.

\section{References}

1. J. Eggleton, S.Radic, and D.J. Moss, Chapter 20, p759-828 in Optical Fiber Telecommunications V: Components and Sub-systems, Edited by Ivan P. Kaminow, Tingye Li, and Alan E. Willner, Academic Press, Oxford, UK, February (2008).

2. Leuthold, C. Koos, and W. Freude, Nat. Photon. 4 (8), 535 (2010). 
3. Li, F. et al., Optics Express 19, 20364-20371 (2011). DOI: 10.1364/OE.19.020364.

4. Li, F. et al., Optics Express 18, 3905-3910 (2010). DOI: 10.1364/OE.18.003905.

5. Ji, et.al., Photonics Technology Letters 221762 (2010).

6. Monat, C. et al., Optics Express 18 (7), 6831-6840 (2010). DOI: 10.1364/OE.18.006831.

7. Corcoran, B., et. al., Optics Express 18, (8) 7770-7781 (2010). DOI: 10.1364/OE.18.007770.

8. G. Ta'eed, et al., Optics Letters $\underline{30} 2900$ (2005). DOl: 10.1364/OL.30.002900.

9. Rochette, J. Kutz, J. Blows, D. Moss, J. T. Mok, and B. J. Eggleton, IEEE Photonics Technology Letters 17908 (2005).

10. Ferrera et al., Optics Express $\underline{22}$ (18) 21488 - 21498 (2014). DOI: 10.1364/OE.22.021488.

11. Monat et al., Nature Communications $\underline{\mathbf{5}}$ Article:3246 (2014). doi:10.1038/ncomms4246.

12. Li, et al., Optics Express 19, (23) 22410-22416 (2011).

13. D. Vo, et al., IEEE Journal of Lightwave Technology $\underline{29}$ (12) 1790-1796 (2011).

14. Ferrera, et al., Optics Express 19 (23) 23153-23161 (2011).

15. Corcoran, et al., Optics Express 1820190 (2010). DOI: 10.1364/OE.18.020190.

16. Caspani, et al., J. of the Optical Society of America B, JOSA B28 (12) A67-A82 (2011).

DOI:10.1364/JOSAB.28.000A67

17. Corcoran, et al., Nature Photonics $\underline{3}$ (4) 206 (2009). doi:10.1038/nphoton.2009.28.

18. J. Moss, H. M. van Driel, and J. E. Sipe, Opt. Lett. 14 (1), 57 (1989).

19. E. Sipe, D. J. Moss, and H. M. van Driel, Phys. Rev. B 35 (3), 1129 (1987).

20. J. Moss, E. Ghahramani, J. E. Sipe, and H. M. van Driel, Phys. Rev. B 41 (3), 1542 (1990).

21. J. Moss, H. M. van Driel, and J. E. Sipe, Appl. Phy. Lett. 4 (17) 1150 (1986).

22. J.Moss, et al., Electronics Letters 41320 (2005). DOI:10.1049/el:20058051

23. R.E. Lamont, et al., Photonics Technology Letters 181185 (2006). DOI:10.1109/LPT.2006.874718.

24. Tuniz, G.Brawley, D.J.Moss, B.J.Eggleton,, Optics Express 1618524 (2008). DOI:

10.1364/OE.16.018524.

25. BJ Eggleton, B Luther-Davies, K Richardson, “Chalcogenide photonics”, Nature Photonics $\underline{\mathbf{5}}$ (3), 141 (2011).

26. Lee, et al., Optics Express 15 1277-1285 (2007). DOI:10.1364/OE.15.001277

27. Tomljenovic-Hanic, M.J. Steel, C. Martijn de Sterke and D. J. Moss, "High-Q cavities in photosensitive photonic crystals", Optics Letters $\underline{32}$ (5) 542-544 (2007). DOI:10.1364/OL.32.000542

28. Grillet, et al., Optics Express 15 (3) 1267-1276 (2007). DOI:10.1364/OE.15.001267

29. G. Ta'eed, N. Baker, L. Fu, K. Finsterbusch, M.R.E. Lamont, H.Nguyen, D.J. Moss, and B.J. Eggleton, Y. Choi, S. Madden, B. Luther-Davies "Ultrafast all-optical chalcogenide glass photonic circuits", Optics Express 159205 (2007). 
30. Freeman, C.Grillet, M.Lee, B.Luther-Davies, CLC Smith, Y Ruan, A Rode, D. J. Moss, and B. J. Eggleton, "Chalcogenide Glass Photonic Crystal Devices”, Photonic and Electromagnetic Crystal Structures, Photonics and Nanostructures-Fundamentals and Applications, Science Direct Elsevier Publishing $\underline{6}$ (1) Pages: 3-11 (2008). doi:10.1016/j.photonics.2007.11.001.

31. Grillet, D.Freeman, B.Luther-Davies, S.Madden, R.McPhedran, D.J. Moss, M.J. Steel, and B.J.Eggleton, "Characterization and modeling of Fano resonances in chalcogenide photonic crystal membranes", Optics Express 14369 (2006).

32. G. Ta'eed, M. Shokooh-Saremi, L.B Fu, D.J. Moss, M.Rochette, I.C.M. Littler, B.J. Eggleton, Y.Ruan and B.Luther-Davies, "Self-phase modulation based integrated optical regeneration in chalcogenide waveguides", IEEE Journal of Selected Topics in Quantum Electronics 12360 (2006).

33. Shokooh-Saremi, V.G. Ta'eed, I.Littler, D.J. Moss and B.J. Eggleton, Y.Ruan and B.Luther-Davies, “High performance Bragg gratings in chalcogenide rib waveguides written with a modified Sagnac interferometer: experiment and modeling", Journal of the Optical Society of America B (JOSA B) $\underline{23}$ 1323 (2006).

34. R.E. Lamont, V.G. Ta'eed, M.A.F. Roelens, D.J. Moss, B.J. Eggleton, DY. Choy, S. Madden and B. LutherDavies, "Error-free wavelength conversion via cross phase modulation in $5 \mathrm{~cm}$ of As2S3 chalcogenide glass rib waveguide”, Electronics Letters $\underline{43} 945$ (2007).

35. [35] Ikeda, K., Saperstein, R. E., Alic, N. \& Fainman, Y., “Thermal and Kerr nonlinear properties of plasma-deposited silicon nitride/silicon dioxide waveguides”, Opt. Express 16 12987-12994 (2008).

36. [36] J. S. Levy, A. Gondarenko, M. A. Foster et al., "CMOS-compatible multiple-wavelength oscillator for on-chip optical interconnects," Nature Photonics 4 (1), 37-40 (2010).

37. [37] L. Razzari, D. Duchesne, M. Ferrera et al., “CMOS-compatible integrated optical hyper-parametric oscillator," Nature Photonics 4(1), 41-45 (2010).

38. [38] D. J. Moss, R. Morandotti, A. L. Gaeta et al., "New CMOS-compatible platforms based on silicon nitride and Hydex for nonlinear optics," Nature Photonics $\underline{7}$ (8), 597-607 (2013).

39. [39] M. Ferrera, L. Razzari, D. Duchesne et al., "Low-power continuous-wave nonlinear optics in doped silica glass integrated waveguide structures," Nature Photonics, $\underline{2}$ (12), 737-740 (2008).

40. [40] M. Ferrera, D. Duchesne, L. Razzari et al., "Low power four wave mixing in an integrated, microring resonator with Q=1.2 million,” Optics Express,17 (16), 14098-14103 (2009).

41. [41] D. Duchesne, M. Peccianti, M. R. E. Lamont et al., "Supercontinuum generation in a high index doped silica glass spiral waveguide," Optics Express,18 (2), 923-930 (2010).

42. [42] M. Ferrera, et al., “On-chip CMOS-compatible all-optical integrator”, Nature Communications, 1 Article 29 (2010). DOI:10.1038/ncomms1028

43. [43] A. Pasquazi, R. Ahmad, M. Rochette et al., "All-optical wavelength conversion in an integrated ring resonator," Optics Express,18 (4), 3858-3863 (2010).

44. [44] A. Pasquazi, Y. Park, J. Azana et al., "Efficient wavelength conversion and net parametric gain via Four Wave Mixing in a high index doped silica waveguide," Optics Express, 18 (8), 7634-7641 (2010). 
45. [45] M. Peccianti, M. Ferrera, L. Razzari et al., "Subpicosecond optical pulse compression via an integrated nonlinear chirper," Optics Express, 18 (8), 7625-7633 (2010).

46. [46] D. Duchesne, M. Ferrera, L. Razzari et al., "Efficient self-phase modulation in low loss, high index doped silica glass integrated waveguides," Optics Express,17 (3), 1865-1870 (2009).

47. [47] Pasquazi, M. Peccianti, L. Razzari, D. J. Moss, S. Coen, M. Erkintalo, Y. K. Chembo, T. Hansson, S. Wabnitz, P. Del'Haye, X. Xue, A. M. Weiner, and R. Morandotti, Physics Reports $\underline{729}$ (1), 1 (2018).

48. [48] P. Del'Haye, A. Schliesser, O. Arcizet, T. Wilken, R. Holzwarth, T.J. Kippenberg, "Optical frequency comb generation from a monolithic microresonator", Nature $\underline{450}$ 1214-1217 (2007).

49. [49] M.Peccianti, A.Pasquazi, Y.Park, B.E Little, S.Chu, D.J Moss, and R.Morandotti, "Demonstration of an ultrafast nonlinear microcavity modelocked laser", Nature Communications, $\underline{3} 765$ (2012). DOI:10.1038/ncomms1762

50. [50] M.Kues, et. al., "Passively modelocked laser with an ultra-narrow spectral width”, Nature Photonics, 11 (3) 159 (2017). DOI:10.1038/nphoton.2016.271

51. [51] A. Pasquazi, L. Caspani, M. Peccianti et al., "Self-locked optical parametric oscillation in a CMOS compatible microring resonator: a route to robust optical frequency comb generation on a chip," Optics Express, 21 (11), 13333-13341 (2013).

52. [52] A. Pasquazi, M. Peccianti, B. E. Little et al., "Stable, dual mode, high repetition rate mode-locked laser based on a microring resonator," Optics Express, $\underline{20}$ (24), 27355-27362 (2012).

53. [53] C. Reimer, L. Caspani, M. Clerici et al., "Integrated frequency comb source of heralded single photons," Optics Express, 22 (6), 6535-6546 (2014).

54. [54] C.Reimer, et al., "Cross-polarized photon-pair generation and bi-chromatically pumped optical parametric oscillation on a chip", Nature Communications, $\underline{6}$ Article 8236 (2015). DOI:

10.1038/ncomms9236

55. [55] L. Caspani, C. Reimer, M. Kues et al., "Multifrequency sources of quantum correlated photon pairs on-chip: a path toward integrated Quantum Frequency Combs," Nanophotonics, $\underline{\mathbf{5}}$ (2), 351-362 (2016).

56. [56] C. Reimer, M. Kues, P. Roztocki et al., "Generation of multiphoton entangled quantum states by means of integrated frequency combs," Science, $\mathbf{3 5 1}$ (6278), 1176-1180 (2016).

57. [57] M.Kues, et al., "On-chip generation of high-dimensional entangled quantum states and their coherent control”, Nature, $\underline{\mathbf{5 4 6}}$ (7660) 622-626 (2017).

58. [58] P. Roztocki, M. Kues, C. Reimer et al., "Practical system for the generation of pulsed quantum frequency combs," Optics Express, $\underline{25}$ (16), 18940-18949 (2017).

59. [59] P.Marin-Palomo, et al., "Microresonator-based solitons for massively parallel coherent optical communications", Nature $\underline{546}$ (7657) 274 (2017).

60. [60] DT Spencer et al., "An optical-frequency synthesizer using integrated photonics", Nature $\underline{\mathbf{5 5 7}}$ (7703) 81-85 (2018). 
61. [61] J Pfeifle, V Brasch, M Lauermann, Y Yu, D Wegner, T Herr, K Hartinger, et al., "Coherent terabit communications with microresonator Kerr frequency combs", Nature Photonics $\underline{8}$ (5), 375-380 (2014).

62. [62] P Del'Haye, T Herr, E Gavartin, ML Gorodetsky, R Holzwarth, et al., "Octave spanning tunable frequency comb from a microresonator”, Physical Review Letters 107 (6), 063901 (2011).

63. [63] TJ Kippenberg, R Holzwarth, SA Diddams, "Microresonator-based optical frequency combs", Science 332 (6029), 555-559 (2011).

64. [64] T Herr, V Brasch, JD Jost, CY Wang, NM Kondratiev, ML Gorodetsky, et al., "Temporal solitons in optical microresonators", Nature Photonics $\underline{8}$ (2), 145-152 (2014).

65. [65] F Ferdous, H Miao, DE Leaird, K Srinivasan, J Wang, L Chen, ... "Spectral line-by-line pulse shaping of on-chip microresonator frequency combs", Nature Photonics $\underline{\mathbf{5}}$ (12), 770 (2011).

66. [66] X Xue, PH Wang, Y Xuan, M Qi, AM Weiner, "Microresonator Kerr frequency combs with high conversion efficiency", Laser \& Photonics Reviews 11 (1) (2017).

67. [67]Y Xuan, Y Liu, LT Varghese, AJ Metcalf, X Xue, PH Wang, K Han, ... "High-Q silicon nitride microresonators exhibiting low-power frequency comb initiation", Optica $\underline{\mathbf{3}}$ (11), 1171-1180 (2016).

68. [68] X Xue, M Qi, AM Weiner, "Normal-dispersion microresonator Kerr frequency combs", Nanophotonics $\underline{\mathbf{5}}$ (2), 244-262 (2016).

69. [69] C.Grillet, et al., "Amorphous Silicon Nanowires with Record High Nonlinearity, FOM, and Optical Stability", Optics Express $\underline{20}$ (20) 22609-22615 (2012). DOI: 10.1364/OE.20.022609.

70. [70] Ju Won Choi, Byoung-Uk Sohn, George F. R. Chen, Doris K. T. Ng, and Dawn T. H. Tan, "Solitoneffect optical pulse compression in CMOS-compatible ultra-silicon-rich nitride waveguides", APL Photonics HIBSP2019, 110804 (2020).

71. Capmany, and D. Novak, "Microwave photonics combines two worlds," Nat. Photonics, vol. 1, no. 6, pp. 319-330, 2007.

72. P. Yao, "Microwave photonics," Journal of Lightwave Technol., vol. 27, no. 1-4, pp. 314-335, Jan-Feb. 2009.

73. Marpaung, J. Yao, and J. Capmany, "Integrated microwave photonics," Nature Photonics, vol. 13, no. 2, pp. 80-90, Feb. 2019.

74. Azaña, "Ultrafast analog all-optical signal processors based on fiber-grating devices," IEEE Photonics Journal, vol. 2, no. 3, pp. 359-386. 2010.

75. Capmany, B. Ortega, and D. Pastor, "A tutorial on microwave photonic filters," Journal of Lightwave Technol., vol. 24, no. 1, pp. 201-229, 2006.

76. R. Supradeepa et al., "Comb-based radiofrequency photonic filters with rapid tunability and high selectivity," Nat. Photonics, vol. 6, no. 3, pp. 186-194, Mar. 2012.

77. Malacarne et al.,"Single-shot photonic time-intensity integration based on a time-spectrum convolution system," Optics Letters, vol. 37, no. 8, pp. 1355-1357, Apr. 2012. 
78. Torres-Company, and A. M. Weiner, "Optical frequency comb technology for ultra-broadband radiofrequency photonics," Laser Photonics Rev, vol. 8, no. 3, pp. 368-393, May. 2014.

79. Jiang, C. B. Huang, D. E. Leaird, and A. M. Weiner, "Optical arbitrary waveform processing of more than 100 spectral comb lines," Nat Photonics, vol. 1, no. 8, pp. 463-467, Aug. 2007.

80. Liu, J. Hotten, A. Choudhary, B. J. Eggleton, and D. Marpaung, "All-optimized integrated RF photonic notch filter," Opt. Lett., vol. 42, no. 22, pp. 4631-4634, Nov 15. 2017.

81. Liu, D. Marpaung, A. Choudhary, J. Hotten, and B. J. Eggleton, “Link performance optimization of chip-based Si3N4 microwave photonic filters," J. Lightwave Technol., to be published. DOI: 10.1109/JLT.2018.2842203.

82. Liu, Y. Yu, S. X. Yuan, X. B. Xu, and X. L. Zhang, "Tunable megahertz bandwidth microwave photonic notch filter based on a silica microsphere cavity," Opt. Lett., vol. 41, no. 21, pp. 5078-5081, Nov 1. 2016.

83. Marpaung, B. Morrison, M. Pagani, R. Pant, D. Y. Choi, B. Luther-Davies, S. J. Madden, and B. J. Eggleton, "Low-power, chip-based stimulated Brillouin scattering microwave photonic filter with ultrahigh selectivity," Optica, vol. 2, no. 2, pp. 76-83, Feb 20. 2015.

84. Choudhary, B. Morrison, I. Aryanfar, S. Shahnia, M. Pagani, Y. Liu, K. Vu, S. Madden, D. Marpaung, and B. J. Eggleton, "Advanced integrated microwave signal processing with giant on-chip Brillouin gain," J. Lightwave Technol., vol. 35, no. 4, pp. 846-854, Feb 15. 2017.

85. Marpaung, B. Morrison, R. Pant, and B. J. Eggleton, "Frequency agile microwave photonic notch filter with anomalously high stopband rejection," Opt. Lett., vol. 38, no. 21, pp. 4300-4303, Nov 1. 2013.

86. Xu, J. Wu, M. Shoeiby, T. G. Nguyen, S. T. Chu, B. E. Little, R. Morandotti, A. Mitchell, and D. J. Moss, "Reconfigurable broadband microwave photonic intensity differentiator based on an integrated optical frequency comb source," APL Photonics, vol. 2, no. 9, Sep. 2017.

87. Q. Zhu, F. Y. Chen, H. F. Peng, and Z. Y. Chen, "Novel programmable microwave photonic filter with arbitrary filtering shape and linear phase," Opt. Express, vol. 25, no. 8, pp. 9232-9243, Apr 17. 2017.

88. Jiang, Y. Yu, H. T. Tang, L. Xu, and X. L. Zhang, "Tunable bandpass microwave photonic filter with ultrahigh stopband attenuation and skirt selectivity," Opt. Express, vol. 24, no. 16, pp. 18655-18663, Aug 8. 2016.

89. Wu, X. Xu, T. G. Nguyen, S. T. Chu, B. E. Little, R. Morandotti, A. Mitchell, and D. J. Moss, “RF Photonics: An Optical Microcombs' Perspective," IEEE J. Sel. Top. Quantum Electron., vol. 24, no. 4, Jul-Aug. 2018.

90. Hamidi, D. E. Leaird, and A. M. Weiner, "Tunable Programmable Microwave Photonic Filters Based on an Optical Frequency Comb," IEEE Journal of Microwave Theory, vol. 58, no. 11, pp. 3269-3278, Nov. 2010.

91. J. Zhu, H. Chi, T. Jin, S. L. Zheng, X. F. Jin, and X. M. Zhang, "All-positive-coefficient microwave photonic filter with rectangular response," Opt. Lett., vol. 42, no. 15, pp. 3012-3015, Aug 1. 2017.

92. Mansoori, and A. Mitchell, "RF transversal filter using an AOTF," IEEE Photonic Tech L, vol. 16, no. 3 , pp. 879-881, Mar. 2004. 
93. S. Leng, W. Zhang, and J. A. R. Williams, "Optimization of superstructured fiber Bragg gratings for microwave photonic filters response," IEEE Photonic Tech L, vol. 16, no. 7, pp. 1736-1738, Jul. 2004.

94. Delgado-Pinar, J. Mora, A. Diez, M. V. Andres, B. Ortega, and J. Capmany, "Tunable and reconfigurable microwave filter by use of a Bragg-grating-based acousto-optic superlattice modulator," Opt. Lett., vol. 30, no. 1, pp. 8-10, Jan 1. 2005.

95. Yu, W. Zhang, and J. A. R. Williams, "High-performance microwave transversal filter using fiber Bragg grating arrays," IEEE Photonic Tech L, vol. 12, no. 9, pp. 1183-1185, Sep. 2000.

96. B. Hunter, R. A. Minasian, and P. A. Krug, "Tunable optical transversal filter based on chirped gratings," Electron. Lett., vol. 31, no. 25, pp. 2205-2207, Dec 7. 1995.

97. Wu, V. R. Supradeepa, C. M. Long, D. E. Leaird, and A. M. Weiner, "Generation of very flat optical frequency combs from continuous-wave lasers using cascaded intensity and phase modulators driven by tailored radio frequency waveforms," Opt. Lett., vol. 35, no. 19, pp. 3234-3236, Oct 1. 2010.

98. Z. Li, and J. P. Yao, "Optical frequency comb generation based on repeated frequency shifting using two Mach-Zehnder modulators and an asymmetric Mach-Zehnder interferometer," Opt. Express, vol. 17, no. 26, pp. 23712-23718, Dec 21. 2009.

99. H. Chen, C. He, D. Zhu, R. H. Guo, F. Z. Zhang, and S. L. Pan, "Generation of a flat optical frequency comb based on a cascaded polarization modulator and phase modulator," Opt. Lett., vol. 38, no. 16, pp. 3137-3139, Aug 15. 2013.

100. Saitoh, M. Kourogi, and M. Ohtsu, "An optical frequency synthesizer using a waveguide-type optical frequency comb generator at 1.5-mu m wavelength," IEEE Photonic Tech $L$, vol. 8, no. 11, pp. 15431545, Nov. 1996.

101. 48 P. del'Haye et al., "Optical frequency comb generation from a monolithic Micro-resonator," Nature, vol. 450, no. 7173, pp. 1214-1217, Dec. 2007.

102. Liang et al., "High spectral purity Kerr frequency comb radio frequency photonic oscillator," Nature communications, vol. 6 pp. 7957. 2015.

103. 36 J. S. Levy et al., "CMOS-compatible multiple-wavelength oscillator for on-chip optical interconnects," Photonics, vol. 4, no. 1, pp. 37-40, Jan 10. 2010.

104. 37 L. Razzari et al., "CMOS-compatible integrated optical hyper-parametric oscillator," Photonics, vol. 4, no. 1, pp. 41-45, Jan 10. 2010.

105. 38 D. J. Moss, R. Morandotti, A. L. Gaeta, and M. Lipson, "New CMOS-compatible platforms based on silicon nitride and Hydex for nonlinear optics," Photonics, vol. 7, no. 8, pp. 597-607, Aug. 2013.

106. J. Kippenberg, A. L. Gaeta, M. Lipson, and M. L. Gorodetsky, "Dissipative Kerr solitons in optical microresonators," Science, vol. 361, no. 6402, pp. eaan8083. 2018.

107. L. Gaeta, M. Lipson, and T. J. Kippenberg, "Photonic-chip-based frequency combs," Nature Photonics, vol. 13, no. 3, pp. 158-169, Mar. 2019.

108. 47 A. Pasquazi et al., "Micro-combs: A novel generation of optical sources," Physics Reports-Review Section of Physics Letters, 729, pp. 1-81, Jan 27. 2018. 
109. C. Cole, E. S. Lamb, P. Del'Haye, S. A. Diddams, and S. B. Papp, “Soliton crystals in Kerr resonators,” Nat. Photonics, vol. 11, no. 10, pp. 671-676, Oct. 2017.

110. Xue et al., "Mode-locked dark pulse Kerr combs in normal-dispersion microresonators," Nature Photonics, vol. 9, no. 9, pp. 594. 2015.

111. Bao et al., “Laser cavity-soliton microcombs,” Nature Photonics, vol. 13, no. 6, pp. 384-389, Jun. 2019.

112. Xue, X. Zheng, and B. Zhou, "Super-efficient temporal solitons in mutually coupled optical cavities," Nature Photonics, May. 2019.

113. Zhou et al., "Soliton bursts and deterministic dissipative Kerr soliton generation in auxiliary-assisted microcavities," Light: Science \& Applications, vol. 8, no. 1, pp. 50, 2019.

114. Stern, X Ji, Y. Okawachi, A.L. Gaeta, M. Lipson, “Battery-operated integrated frequency comb generator", Nature vol.562 (7727), 401 (2018).

115. 39 M. Ferrera, L. Razzari, D. Duchesne, R. Morandotti, Z. Yang, M. Liscidini, J. E. Sipe, S. Chu, B. E. Little, and D. J. Moss, “Low-power continuous-wave nonlinear optics in doped silica glass integrated waveguide structures," Nature Photonics, vol. 2, no. 12, pp. 737-740, Dec. 2008.

116. 49 Peccianti, A. Pasquazi, Y. Park, B. E. Little, S. T. Chu, D. J. Moss, and R. Morandotti, “Demonstration of a stable ultrafast laser based on a nonlinear microcavity," Nat. Commun., vol. 3, no. 765, pp. 1-6, Apr. 2012.

117. 52 A. Pasquazi, M. Peccianti, B. E. Little, S. T. Chu, D. J. Moss, and R. Morandotti, "Stable, dual mode, high repetition rate mode-locked laser based on a microring resonator," Opt. Exp., vol. 20, no. 24, pp. 27355-27362, Nov. 2012.

118. 51 A. Pasquazi, L. Caspani, M. Peccianti, M. Clerici, M. Ferrera, L. Razzari, D. Duchesne, B. E. Little, S. T. Chu, D. J. Moss, and R. Morandotti, "Self-locked optical parametric oscillation in a CMOS compatible microring resonator: a route to robust optical frequency comb generation on a chip," Opt. Exp., vol. 21, no. 11, pp. 13333-13341, Jun. 2013.

119. 44 A. Pasquzai, Y. Park, J. Azana, F. Legare, R. Morandotti, B. E. Little, S. T. Chu, and D. J. Moss, "Efficient wavelength conversion and net parametric gain via Four wave Mixing in a high index doped silica waveguide," Opt. Exp., vol. 18, no. 8, pp. 7634-7641, Apr. 2010.

120. 45 M. Peccianti, M. Ferrera, L. Razzari, R. Morandotti, B. E. Little, S. T. Chu, and D. J. Moss, "Subpicosecond optical pulse compression via an integrated nonlinear chirper," Opt. Exp., vol. 18, no. 8, pp. 7625-7633, Apr. 2010.

121. 41 Duchesne, M. Peccianti, M. R. E. Lamont, M. Ferrera, L. Razzari, F. Legare, R. Morandotti, S. Chu, B. E. Little, and D. J. Moss, "Supercontinuum generation in a high index doped silica glass spiral waveguide," Opt. Exp., vol. 18, no. 2, pp. 923-930, Jan. 2010.

122. Bao, L.Olivieri, M.Rowley, S.T. Chu, B.E. Little, R.Morandotti, D.J. Moss, J.S.T. Gongora, M.Peccianti and A.Pasquazi, "Turing patterns in a fibre laser with a nested micro-resonator: robust and controllable micro-comb generation”, in press, Physical Review X (2019). 
123. D. Lauro, J. Li, D. J. Moss, R. Morandotti, S. T. Chu, M. Peccianti, and A. Pasquazi, “Parametric control of thermal self-pulsation in micro-cavities," Opt. Lett. vol. 42, no. 17, pp. 3407-3410, Aug. 2017.

124. Bao, A. Cooper, S. T. Chu, D. J. Moss, R. Morandotti, B. E. Little, M. Peccianti, and A. Pasquazi, "Type-II micro-comb generation in a filter-driven four wave mixing laser," Photonics Research, vol. 6, no. 5, pp. B67-B73, May 2018.

125. Wang, Z. Lu, W. Zhang, S. T. Chu, B. E. Little, L. Wang, X. Xie, M. Liu, Q. Yang, L. Wang, J. Zhao, G. Wang, Q. Sun, Y. Liu, Y. Wang, and W. Zhao, "Robust soliton crystals in a thermally controlled microresonator," Opt. Lett. 43(9), 2002-2005 (2018).

126. Xingyuan Xu, Mengxi Tan, Jiayang Wu, Andreas Boes, Bill Corcoran, Thach G. Nguyen, Sai T. Chu, Brent E. Little, Roberto Morandotti, Arnan Mitchell, and David J. Moss, “Photonic RF phase-encoded signal generation with a microcomb source”, IEEE Journal of Lightwave Technology 38 (2020). DOI:10.1109/JLT.2019.2958564.

127. Mengxi Tan, Xingyuan Xu, Bill Corcoran, Jiayang Wu, Andy Boes, Thach G. Nguyen, Sai T. Chu, Brent E. Little, Roberto Morandotti, Arnan Mitchell, and David J. Moss, "Broadband microwave and RF photonic fractional Hilbert transformer based on a $50 \mathrm{GHz}$ integrated Kerr micro-comb”, IEEE Journal of Lightwave Technology $\underline{37}$ (24) 6097 - 6104 (2019). DOI:10.1109/JLT.2019.2946606

128. Xu, J.Wu, Mengxi Tan, T.G. Nguyen, S.T. Chu, B. E. Little, R.Morandotti, A.Mitchell, and David J. Moss, "Broadband microwave frequency conversion based on an integrated optical micro-comb source", IEEE Journal of Lightwave Technology 37 (2019). IEEE/OSA Journal of Lightwave Technology, Digital Object Identifier: 10.1109/JLT.2019.2930466

129. Xingyuan Xu, Mengxi Tan, Jiayang Wu, Roberto Morandotti, Arnan Mitchell, and David J. Moss, “Microcomb-based photonic RF signal processing”, IEEE Photonics Technology Letters 31 (2019). DOI: 10.1109/LPT.2019.2940497

130. Xu et al., "Photonic microwave true time delays for phased array antennas using a $49 \mathrm{GHz}$ FSR integrated optical micro-comb source," Photonics Res, vol. 6, no. 5, pp. B30-B36, 2018.

131. Xue et al., "Microcomb-based true-time-delay network for microwave beamforming with arbitrary beam pattern control," Journal of Lightwave Technology, vol. 36, no. 12, pp. 2312-2321, Jun. 2018.

132. Xu et al., "Advanced RF and microwave functions based on an integrated optical frequency comb source," Opt. Express, vol. 26, no. 3, pp. 2569-2583, Feb. 2018.

133. Xue, et al., "Programmable single-bandpass photonic RF filter based on a Kerr comb from a microring," Journal of Lightwave Technol., vol. 32, no. 20, pp. 3557-3565, Oct. 2014.

134. Xu et al., "Advanced adaptive photonic RF filters with 80 taps based on an integrated optical microcomb source," Journal of Lightwave Technology, vol. 37, no. 4, pp. 1288-1295, 2019/02/15. 2019.

135. Xu et al., "Reconfigurable broadband microwave photonic intensity differentiator based on an integrated optical frequency comb source," APL Photonics, vol. 2, no. 9, Sep. 2017.

136. G. Nguyen et al., "Integrated frequency comb source-based Hilbert transformer for wideband microwave photonic phase analysis," Opt. Express, vol. 23, no. 17, pp. 22087-22097, Aug. 2015. 
137. Xu et al., "Broadband RF channelizer based on an integrated optical frequency Kerr comb source," Journal of Lightwave Technology, vol. 36, no. 19, pp. 7, 2018. 2018.

138. Xu et al., "High performance RF filters via bandwidth scaling with Kerr micro-combs," APL Photonics, vol. 4, no. 2, pp. 026102. 2019.

139. Wu et al., "RF photonics: An optical microcombs' perspective," IEEE J. Sel. Top. Quantum Electron., vol. 24, no. 4, Jul-Aug. 2018.

140. Xu et al., "Continuously tunable orthogonally polarized RF optical single sideband generator based on micro-ring resonators," Journal of Optics, vol. 20, no. 11, pp. 115701. 2018.

141. Xu et al., "Orthogonally polarized RF optical single sideband generation and dual-channel equalization based on an integrated microring resonator," Journal of Lightwave Technology, vol. 36, no. 20, pp. 4808-4818. 2018.

142. Mengxi Tan, Xingyuan Xu, Bill Corcoran, Jiayang Wu, Andy Boes, Thach G. Nguyen, Sai T. Chu, Brent E. Little, Roberto Morandotti, Arnan Mitchell, and David J. Moss, "Broadband microwave and RF fractional differentiator using photonics", submitted, IEEE Transactions on Circuits and Systems 37 (?) (2019).

143. J. Metcalf et al., "Integrated line-by-line optical pulse shaper for high-fidelity and rapidly reconfigurable RF-filtering," Optics Express, vol. 24, no. 21, pp. 23925-23940. 2016.

144. McClellan, T. W. Parks, and L. Rabiner, "A computer program for designing optimum FIR linear phase digital filters," Transactions on Audio and Electroacoustics, vol. 21, no. 6, pp. 506-526, Dec, 1973.

145. Wu, X. Xu, T. G. Nguyen, S. T. Chu, B. E. Little, R. Morandotti, A. Mitchell, and D. J. Moss, "RF Photonics: An Optical Microcombs' Perspective," IEEE Journal of Selected Topics in Quantum Electronics, 24, no. 4, pp. 1-20. 2018.

146. Xu, M. Tan, J. Wu, R. Morandotti, A. Mitchell, and D. J. Moss, "Microcomb-based photonic RF signal processing," IEEE Photonics Technology Letters, 31, no. 23, pp. 1854-1857. 2019.

147. Xu, J. Wu, T. G. Nguyen, T. Moein, S. T. Chu, B. E. Little, R. Morandotti, A. Mitchell, and D. J. Moss, "Photonic microwave true time delays for phased array antennas using a $49 \mathrm{GHz}$ FSR integrated optical micro-comb source [Invited]," Photonics Research, 6, no. 5, pp. B30-B36, May 1. 2018.

148. Xu, J. Wu, T. G. Nguyen, M. Shoeiby, S. T. Chu, B. E. Little, R. Morandotti, A. Mitchell, and D. J. Moss, "Advanced RF and microwave functions based on an integrated optical frequency comb source," Optics Express, 26, no. 3, pp. 2569-2583, Feb 5. 2018.

149. Xu, M. Tan, J. Wu, T. G. Nguyen, S. T. Chu, B. E. Little, R. Morandotti, A. Mitchell, and D. J. Moss, "Advanced Adaptive Photonic RF Filters with 80 Taps based on an integrated optical micro-comb," Journal of Lightwave Technology, 37, no. 4, pp. 1288-1295, 2019.

150. Xu, J. Wu, M. Shoeiby, T. G. Nguyen, S. T. Chu, B. E. Little, R. Morandotti, A. Mitchell, and D. J. Moss, "Reconfigurable broadband microwave photonic intensity differentiator based on an integrated optical frequency comb source," APL Photonics, 2, no. 9, Sep. 2017.

151. Tan, X. Xu, B. Corcoran, J.Wu, A. Boes, T. G. Nguyen, S.T. Chu, B.E. Little, R. Morandotti, A. Mitchell, and D. J. Moss, "Microwave and RF photonic fractional Hilbert transformer based on a $50 \mathrm{GHz}$ Kerr 
microcomb," J. of Lightwave Technology, 37, no. 24, p 6097, 2019.

152. Tan, et al., "RF and microwave fractional differentiator based on photonics", IEEE Transactions on Circuits and Systems: Express Briefs, Vol. 67, Issue: 11, pp.2767-2771 (2020).

DOI:10.1109/TCSII.2020.2965158.

153. Xu, J. Wu, M. Tan, T. G. Nguyen, S. Chu, B. Little, R. Morandotti, A. Mitchell, and D. J. Moss, "Microcomb based photonic local oscillator for broadband microwave frequency conversion," Journal of Lightwave Technology, 38, no. 2, pp. 332-338. 2020.

154. Xu, et al., "Photonic RF phase-encoded signal generation with a microcomb source", Journal of Lightwave Technology, vol. 38, no. 7, pp. 1722-1727, 2020. DOI: 10.1109/JLT.2019.2958564

155. Xu, M. Tan, J. Wu, T. G. Nguyen, S. T. Chu, B. E. Little, R. Morandotti, A. Mitchell, and D. J. Moss, “High performance RF filters via bandwidth scaling with Kerr micro-combs," APL Photonics, vol. 4, no. 2, pp. 026102. 2019.

156. Xu, J. Wu, T. G. Nguyen, S. Chu, B. Little, A. Mitchell, R. Morandotti, and D. J. Moss, "Broadband RF Channelizer based on an Integrated Optical Frequency Kerr Comb Source," Journal of Lightwave Technology, vol. 36, no. 19, pp. 7, 2018.

157. Xu, J.Wu, T.G. Nguyen, S.T. Chu, B.E. Little, R.Morandotti, A.Mitchell, and D. J. Moss, “Continuously tunable orthogonally polarized optical RF single sideband generator and equalizer based on an integrated microring resonator", IOP Journal of Optics, vol. 20 no.11, p115701, 2018.

158. Xu, J.Wu, T.G.Nguyen, S.T.Chu, B.E.Little, R.Morandotti, A.Mitchell, and D.J. Moss, “Orthogonally polarized optical RF single sideband generator and equalizer based on an integrated micro-ring resonator", Journal of Lightwave Technology Vol. 36, No. 20, 4808-4818 (2018).

159. Bao, H., et al., Laser Cavity Soliton Micro-Combs, Nat. Photon., vol.13, pp.384-389 (2019).

160. Corcoran, et al., "Ultra-dense optical data transmission over standard fiber with a single chip source”, Nature Communications, vol. 11, Article:2568, 2020. DOI:10.1038/s41467-020-16265-x.

161. Xu, et al., "Photonic perceptron based on a Kerr microcomb for scalable high speed optical neural networks", Laser and Photonics Reviews, vol. 14, no. 8, 2000070, 2020. DOI:10.1002/Ipor.202000070.

162. Xu, et al., "11 TOPs photonic convolutional accelerator for optical neural networks", Nature, vol.589 (7840) 44-51 (2021). DOI: 10.1038/s41586-020-03063-0.

163. Xu et al., "11 TeraFLOPs per second photonic convolutional accelerator for deep learning optical neural networks", arXiv preprint arXiv:2011.07393 (2020).

164. Moss, "11 Tera-FLOP/s photonic convolutional accelerator and deep learning optical neural networks", Research Square (2021). DOI: https://doi.org/10.21203/rs.3.rs-493347/v1 .

165. Moss, "11.0 Tera-FLOP/second photonic convolutional accelerator for deep learning optical neural networks", TechRxiv. Preprint (2020). https://doi.org/10.36227/techrxiv.13238423.v1.

166. Moss, David. "11 Tera-flop/s Photonic Convolutional Accelerator for Optical Neural Networks." OSF Preprints, 23 Feb. (2021). DOI: 10.31219/osf.io/vqt4s. 
167. Mengxi Tan, X. Xu, J. Wu, T. G. Nguyen, S. T. Chu, B. E. Little, R. Morandotti, A. Mitchell, and David J. Moss, "Photonic Radio Frequency Channelizers based on Kerr Optical Micro-combs", Journal of Semiconductors, Vol. 42, No. 4, 041302 (2021). (ISSN 1674-4926). DOI:10.1088/16744926/42/4/041302.

168. Bao, L.Olivieri, M.Rowley, S.T. Chu, B.E. Little, R.Morandotti, D.J. Moss, J.S.T. Gongora, M.Peccianti and A. Pasquazi, "Laser Cavity Solitons and Turing Patterns in Microresonator Filtered Lasers: properties and perspectives", Paper No. LA203-5, Paper No. 11672-5, SPIE LASE, SPIE Photonics West, San Francisco CA March 6-11 (2021). DOI:10.1117/12.2576645

169. Mengxi Tan, X. Xu, J. Wu, A. Boes, T. G. Nguyen, S. T. Chu, B. E. Little, R. Morandotti, A. Mitchell, and David J. Moss, "Advanced microwave signal generation and processing with soliton crystal microcombs", or "Photonic convolutional accelerator and neural network in the Tera-OPs regime based on Kerr microcombs", Paper No. 11689-38, PW210-OE201-67, Integrated Optics: Devices, Materials, and Technologies XXV, SPIE Photonics West, San Francisco CA March 6-11 (2021). DOI: $10.1117 / 12.2584017$

170. Mengxi Tan, Bill Corcoran, Xingyuan Xu, Andrew Boes, Jiayang Wu, Thach Nguyen, Sai T. Chu, Brent E. Little, Roberto Morandotti, Arnan Mitchell, and David J. Moss, "Optical data transmission at 40 Terabits/s with a Kerr soliton crystal microcomb", Paper No.11713-8, PW210-0E803-23, NextGeneration Optical Communication: Components, Sub-Systems, and Systems X, SPIE Photonics West, San Francisco CA March 6-11 (2021). DOI:10.1117/12.2584014

171. Mengxi Tan, X. Xu, J. Wu, A. Boes, T. G. Nguyen, S. T. Chu, B. E. Little, R. Morandotti, A. Mitchell, and David J. Moss, "RF and microwave photonic, fractional differentiation, integration, and Hilbert transforms based on Kerr micro-combs", Paper No. 11713-16, PW210-OE803-24, Next-Generation Optical Communication: Components, Sub-Systems, and Systems X, SPIE Photonics West, San Francisco CA March 6-11 (2021). DOI:10.1117/12.2584018

172. Mengxi Tan, X. Xu, J. Wu, A. Boes, T. G. Nguyen, S. T. Chu, B. E. Little, R. Morandotti, A. Mitchell, and David J. Moss, "Broadband photonic RF channelizer with 90 channels based on a soliton crystal microcomb", or "Photonic microwave and RF channelizers based on Kerr micro-combs", Paper No. 11685-22, PW210-OE106-49, Terahertz, RF, Millimeter, and Submillimeter-Wave Technology and Applications XIV, SPIE Photonics West, San Francisco CA March 6-11 (2021).

DOI:10.1117/12.2584015

173. Xu, M. Tan, J. Wu, S. T. Chu, B. E. Little, R. Morandotti, A. Mitchell, B. Corcoran, D. Hicks, and D. J. Moss, "Photonic perceptron based on a Kerr microcomb for scalable high speed optical neural networks", IEEE Topical Meeting on Microwave Photonics (MPW), pp. 220-224, Matsue, Japan, November 24-26, 2020. Electronic ISBN:978-4-88552-331-1. DOI: 10.23919/MWP48676.2020.9314409

174. Mengxi Tan, Bill Corcoran, Xingyuan Xu, Andrew Boes, Jiayang Wu, Thach Nguyen, S.T. Chu, B. E. Little, Roberto Morandotti, Arnan Mitchell, and David J. Moss, "Ultra-high bandwidth optical data transmission with a microcomb", IEEE Topical Meeting on Microwave Photonics (MPW), pp. 78-82. 
Virtual Conf., Matsue, Japan, November 24-26, 2020. Electronic ISBN:978-4-88552-331-1. DOI: 10.23919/MWP48676.2020.9314476

175. Tan, X. Xu, J. Wu, R. Morandotti, A. Mitchell, and D. J. Moss, "RF and microwave high bandwidth signal processing based on Kerr Micro-combs", Advances in Physics X, VOL. 6, NO. 1, 1838946 (2020). DOI:10.1080/23746149.2020.1838946.

176. Tan, X. Xu, J. Wu, D.J. Moss, “High bandwidth temporal RF photonic signal processing with Kerr micro-combs: integration, fractional differentiation and Hilbert transforms", arXiv preprint arXiv:2103.03674 (2021).

177. Moss, "Temporal RF photonic signal processing with Kerr micro-combs: Hilbert transforms, integration and fractional differentiation”, OSF Preprints, 18 Feb. (2021). DOI: 10.31219/osf.io/hx9gb.

178. Moss, "RF and microwave photonic high bandwidth signal processing based on Kerr micro-comb sources”, TechRxiv. Preprint (2020). DOI:10.36227/techrxiv.12665609.v3.

179. Moss, "RF and microwave photonic signal processing with Kerr micro-combs”, Research Square (2021). DOI: 10.21203/rs.3.rs-473364/v1.

180. Tan, X. Xu, J. Wu, D.J. Moss, “RF Photonic Signal Processing with Kerr Micro-Combs: Integration, Fractional Differentiation and Hilbert Transforms", Preprints (2020). 2020090597. doi:10.20944/preprints202009.0597.v1.

181. Mengxi Tan, Xingyuan Xu, Jiayang Wu, Thach G. Nguyen, Sai T. Chu, Brent E. Little, Roberto Morandotti, Arnan Mitchell, and David J. Moss, “Photonic Radio Frequency Channelizers based on Kerr Micro-combs and Integrated Micro-ring Resonators", JOSarXiv.202010.0002.

182. Mengxi Tan, Xingyuan Xu, David Moss “Tunable Broadband RF Photonic Fractional Hilbert Transformer Based on a Soliton Crystal Microcomb", Preprints, DOI: 10.20944/preprints202104.0162.v1

183. Mengxi Tan, X. Xu, J. Wu, T. G. Nguyen, S. T. Chu, B. E. Little, R. Morandotti, A. Mitchell, and David J. Moss, "Orthogonally polarized Photonic Radio Frequency single sideband generation with integrated micro-ring resonators", Journal of Semiconductors vol. 42, No.4, 041305 (2021). DOI: 10.1088/16744926/42/4/041305.

184. Mengxi Tan, X. Xu, J. Wu, T. G. Nguyen, S. T. Chu, B. E. Little, R. Morandotti, A. Mitchell, and David J. Moss, "Photonic Radio Frequency Channelizers based on Kerr Optical Micro-combs”, Journal of Semiconductors 42 (4), 041302 (2021). (ISSN 1674-4926). DOI:10.1088/1674-4926/42/4/041302.

185. Mengxi Tan, Xingyuan Xu, David Moss “Tunable Broadband RF Photonic Fractional Hilbert Transformer Based on a Soliton Crystal Microcomb”, Preprints, DOI: 10.20944/preprints202104.0162.v1

186. Mengxi Tan, X. Xu, J. Wu, T. G. Nguyen, S. T. Chu, B. E. Little, R. Morandotti, A. Mitchell, and David J. Moss, "Orthogonally polarized Photonic Radio Frequency single sideband generation with integrated micro-ring resonators", Journal of Semiconductors 42 (4), 041305 (2021). DOI: 10.1088/16744926/42/4/041305. 
187. Razzari, D. Duchesne, M. Ferrera, et al., "CMOS-compatible integrated optical hyper-parametric oscillator," Nature Photonics, vol. 4, no. 1, pp. 41-45 (2010).

188. Ferrera, L. Razzari, D. Duchesne, et al., "Low-power continuous-wave nonlinear optics in doped silica glass integrated waveguide structures," Nature Photonics, vol. 2, no. 12, pp. 737-740 (2008).

189. Pasquazi, et al., "Sub-picosecond phase-sensitive optical pulse characterization on a chip", Nature Photonics, vol. 5, no. 10, pp. 618-623 (2011). DOI: 10.1038/nphoton.2011.199.

190. Duchesne, M. Peccianti, M. R. E. Lamont, et al., "Supercontinuum generation in a high index doped silica glass spiral waveguide," Optics Express, vol. 18, no, 2, pp. 923-930 (2010).

191. Ferrera, et al., "On-chip CMOS-compatible all-optical integrator”, Nature Communications, vol. 1, Article 29 (2010).

192. Bao et al., "Turing patterns in a fibre laser with a nested micro-resonator: robust and controllable micro-comb generation", Physical Review Research, vol. 2, pp. 023395 (2020).

193. D. Lauro, J. Li, D. J. Moss, R. Morandotti, S. T. Chu, M. Peccianti, and A. Pasquazi, "Parametric control of thermal self-pulsation in micro-cavities," Opt. Lett. vol. 42, no. 17, pp. 3407-3410, Aug. 2017.

194. Bao et al., "Type-II micro-comb generation in a filter-driven four wave mixing laser," Photonics Research, vol. 6, no. 5, pp. B67-B73 (2018).

195. Pasquazi, et al., "All-optical wavelength conversion in an integrated ring resonator," Optics Express, vol. 18, no. 4, pp. 3858-3863 (2010).

196. Pasquazi, Y. Park, J. Azana, et al., "Efficient wavelength conversion and net parametric gain via Four Wave Mixing in a high index doped silica waveguide," Optics Express, vol. 18, no. 8, pp. 7634-7641 (2010).

197. Peccianti, M. Ferrera, L. Razzari, et al., "Subpicosecond optical pulse compression via an integrated nonlinear chirper," Optics Express, vol. 18, no. 8, pp. 7625-7633 (2010).

198. Duchesne, M. Ferrera, L. Razzari, et al., "Efficient self-phase modulation in low loss, high index doped silica glass integrated waveguides," Optics Express, vol. 17, no. 3, pp. 1865-1870 (2009).

199. Peccianti, et al., "Demonstration of an ultrafast nonlinear microcavity modelocked laser", Nature Communications, vol. 3, pp. 765 (2012).

200. Kues, et al., "Passively modelocked laser with an ultra-narrow spectral width", Nature Photonics, vol. 11, no. 3, pp. 159 (2017). DOI:10.1038/nphoton.2016.271

201. Pasquazi, L. Caspani, M. Peccianti, et al., "Self-locked optical parametric oscillation in a CMOS compatible microring resonator: a route to robust optical frequency comb generation on a chip," Optics Express, vol. 21, no. 11, pp. 13333-13341 (2013).

202. Pasquazi, M. Peccianti, B. E. Little, et al., "Stable, dual mode, high repetition rate mode-locked laser based on a microring resonator," Optics Express, vol. 20, no. 24, pp. 27355-27362 (2012).

203. Reimer, L. Caspani, M. Clerici, et al., "Integrated frequency comb source of heralded single photons," Optics Express, vol. 22, no. 6, pp. 6535-6546 (2014). 
204. Reimer, et al., "Cross-polarized photon-pair generation and bi-chromatically pumped optical parametric oscillation on a chip", Nature Communications, vol. 6, Article 8236 (2015). DOI: 10.1038/ncomms9236

205. Caspani, C. Reimer, M. Kues, et al., "Multifrequency sources of quantum correlated photon pairs onchip: a path toward integrated Quantum Frequency Combs," Nanophotonics, vol. 5, no. 2, pp. 351-362 (2016).

206. Reimer, M. Kues, P. Roztocki, B. Wetzel, F. Grazioso, B. E. Little, S. T. Chu, T. Johnston, Y. Bromberg, L. Caspani, D. J. Moss, and R. Morandotti, "Generation of multiphoton entangled quantum states by means of integrated frequency combs," Science, vol. 351, no. 6278, pp. 1176-1180 (2016).

207. Roztocki, M. Kues, C. Reimer, B. Wetzel, S. Sciara, Y. Zhang, A. Cino, B. E. Little, S. T. Chu, D. J. Moss, and R. Morandotti, "Practical system for the generation of pulsed quantum frequency combs," Optics Express, vol. 25, no. 16, pp. 18940-18949 (2017).

208. Zhang, et al., "Induced photon correlations through superposition of two four-wave mixing processes in integrated cavities", Laser and Photonics Reviews, vol. 14, no. 7, 2000128 (2020). DOI: 10.1002/Ipor.202000128

209. Kues, C. Reimer, A. Weiner, J. Lukens, W. Munro, D. J. Moss, and R. Morandotti, "Quantum Optical Micro-combs", Nature Photonics, vol. 13, no.3, pp. 170-179 (2019).

210. Reimer, et al.,"High-dimensional one-way quantum processing implemented on d-level cluster states”, Nature Physics, vol. 15, no.2, pp. 148-153 (2019).

211. Ido, H.Sano, D.J.Moss, S.Tanaka, and A.Takai, "Strained InGaAs/InAIAs MQW electroabsorption modulators with large bandwidth and low driving voltage", Photonics Technology Letters, Vol. 6 , 1207 (1994). DOI: 10.1109/68.329640.

212. Arianfard et al., "Three waveguide coupled sagnac loop reflectors for advanced spectral engineering," J. Lightwave Technol., doi: 10.1109/JLT.2021.3066256.

213. Wu et al., "Nested configuration of silicon microring resonator with multiple coupling regimes," IEEE Photon. Technol. Lett., vol. 25, no. 6, pp. 580-583, Mar. 2013.

214. Wu, T. Moein, X. Xu, and D. J. Moss, "Advanced photonic filters based on cascaded Sagnac loop reflector resonators in silicon-on-insulator nanowires," APL Photonics, vol. 3, 046102 (2018). DOI:/10.1063/1.5025833Apr. 2018.

215. Wu, T. Moein, X. Xu, D.J. Moss, “Silicon photonic filters based on cascaded Sagnac loop resonators", arXiv preprint arXiv:1805.05405a (2018).

216. Wu, T. Moein, X. Xu, G. H. Ren, A. Mitchell, and D. J. Moss, "Micro-ring resonator quality factor enhancement via an integrated Fabry-Perot cavity," APL Photonics, vol. 2, 056103 (2017).

217. Arianfard, J. Wu, S. Juodkazis, and D. J. Moss, "Advanced Multi-Functional Integrated Photonic Filters Based on Coupled Sagnac Loop Reflectors", Journal of Lightwave Technology, Vol. 39, No.5, pp.1400-1408 (2021). DOI: 10.1109/JLT.2020.3037559.

218. J. Moss, "Optimization of Optical Filters based on Integrated Coupled Sagnac Loop Reflectors", Research Square (2021). DOI: https://doi.org/10.21203/rs.3.rs-478204/v1 
219. Arianfard, J. Wu, S. Juodkazis, D. J. Moss, "Spectral Shaping Based on Integrated Coupled Sagnac Loop Reflectors Formed by a Self-Coupled Wire Waveguide", submitted, IEEE Photonics Technology Letters, vol. 33 (2021).

220. Wu et al., "Graphene oxide waveguide and micro-ring resonator polarizers," Laser Photonics Rev., vol. 13, no. 9, pp. 1900056, Aug. 2019.

221. Zhang et al., "Optimizing the Kerr nonlinear optical performance of silicon waveguides integrated with 2D graphene oxide films," J. Lightwave Technol., doi: 10.1109/JLT.2021.3069733.

222. Qu et al., "Analysis of four-wave mixing in silicon nitride waveguides integrated with 2D layered graphene oxide films," J. Lightwave Technol., vol. 39, no. 9, pp. 2902-2910, May. 2021.

223. Qu et al., "Enhanced four-wave mixing in silicon nitride waveguides integrated with 2D layered graphene oxide films," Adv. Opt. Mater., vol. 8, no. 20, pp. 2001048, Oct. 2020.

224. Wu et al., " $2 \mathrm{D}$ layered graphene oxide films integrated with micro-ring resonators for enhanced nonlinear optics," Small, vol. 16, no. 16, pp. 1906563, Mar. 2020.

225. Moss, David; Wu, Jiayang; xu, xingyuan; Yang, Yunyi; jia, linnan; Zhang, Yuning; et al. (2020): Enhanced optical four-wave-mixing in integrated ring resonators with graphene oxide films. TechRxiv. Preprint. https://doi.org/10.36227/techrxiv.11859429.v1.

226. Wu, J.; Yang, Y.; Qu, Y.; Jia, L.; Zhang, Y.; Xu, X.; Chu, S.T.; Little, B.E.; Morandotti, R.; Jia, B.; Moss, D.J. Enhancing Third Order Nonlinear Optics in Integrated Ring Resonators with 2D Material Films. Preprints 2020, 2020030107

227. Wu, J.; Yang, Y.; Qu, Y.; Jia, L.; Zhang, Y.; Xu, X.; Chu, S.T.; Little, B.E.; Morandotti, R.; Jia, B.; Moss, D.J. Enhancing Third Order Nonlinear Optics in Integrated Ring Resonators with 2D Material Films. Preprints 2020, 2020030107

228. Wu et al., "Enhanced four-wave-mixing with $2 \mathrm{D}$ layered graphene oxide films integrated with CMOS compatible micro-ring resonators", arXiv preprint, arXiv:2002.04158 (2020).

229. Zhang et al., "Enhanced Kerr nonlinearity and nonlinear figure of merit in silicon nanowires integrated with 2D graphene oxide films," ACS Appl. Mater. Interfaces, vol. 12, no. 29, pp. 33094-33103, Jun. (2020).

230. Zhang et al., "Enhanced nonlinear optical figure-of-merit at $1550 \mathrm{~nm}$ for silicon nanowires integrated with graphene oxide layered films", arXiv preprint arXiv:2004.08043 (2020).

231. Moss et al., "Transforming silicon into a high performing integrated nonlinear photonics platform by integration with 2D graphene oxide films", TechRxiv. Preprint. (2020).

https://doi.org/10.36227/techrxiv.12061809.v1.

232. Moss, "Elevating silicon into a high performance nonlinear optical platform through the integration of 2D graphene oxide thin films", Research Square (2021). DOI: https://doi.org/10.21203/rs.3.rs$511259 / \mathrm{v} 1$.

233. Moss, D.; Wu, J.; Jia, B.; Yang, Y.; Qu, Y.; Jia, L.; Zhang, Y. “Improved Nonlinear Optics in Silicon-oninsulator Nanowires Integrated with 2D Graphene Oxide Films", Preprints (2020), 2020040033. 
234. Yang et al., "Invited article: enhanced four-wave mixing in waveguides integrated with graphene oxide," APL Photonics, vol. 3, no. 12, pp. 120803, Oct. 2018.

235. T.D. Vo et al., "Silicon-chip-based real-time dispersion monitoring for $640 \mathrm{Gbit} / \mathrm{s}$ DPSK signals", Journal of Lightwave Technology, vol. 29, no. 12, 1790-1796 (2011).

\section{Figures}
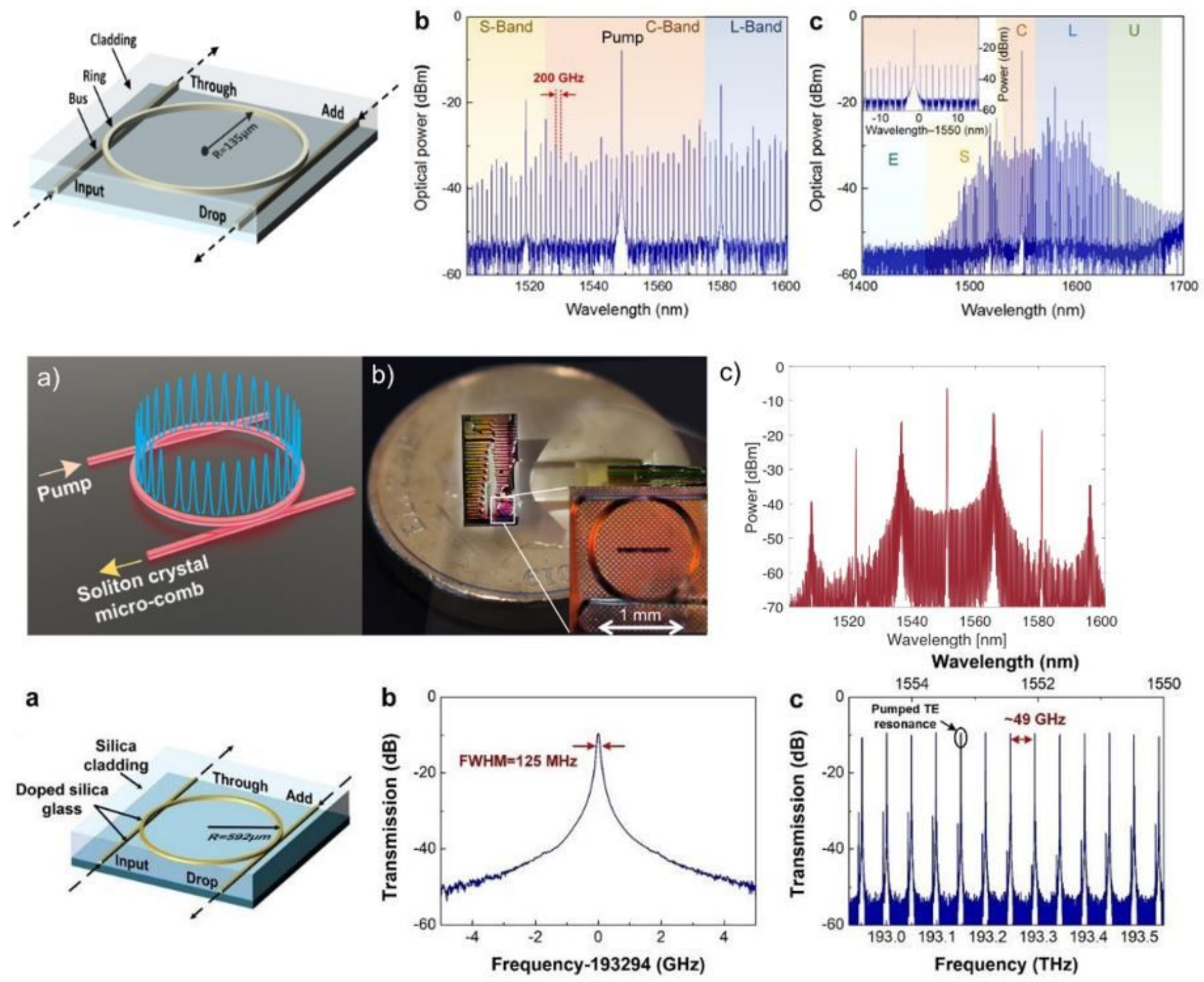

\section{Figure 1}

Schematic illustration of the integrated MRRs for generating the Kerr micro-comb for both $200 \mathrm{GHz} F S R$ combs (a-c) and $49 \mathrm{GHz}$ combs (d-h). (b) A resonance at $193.294 \mathrm{THz}$ with full width at half maximum (FWHM) of $124.94 \mathrm{MHz}$, corresponding to a quality factor of $1.549 \times 106$. (c) Drop-port transmission spectrum of the integrated MRR with a span of $5 \mathrm{~nm}$, showing an optical free spectral range of $49 \mathrm{GHz}$. 

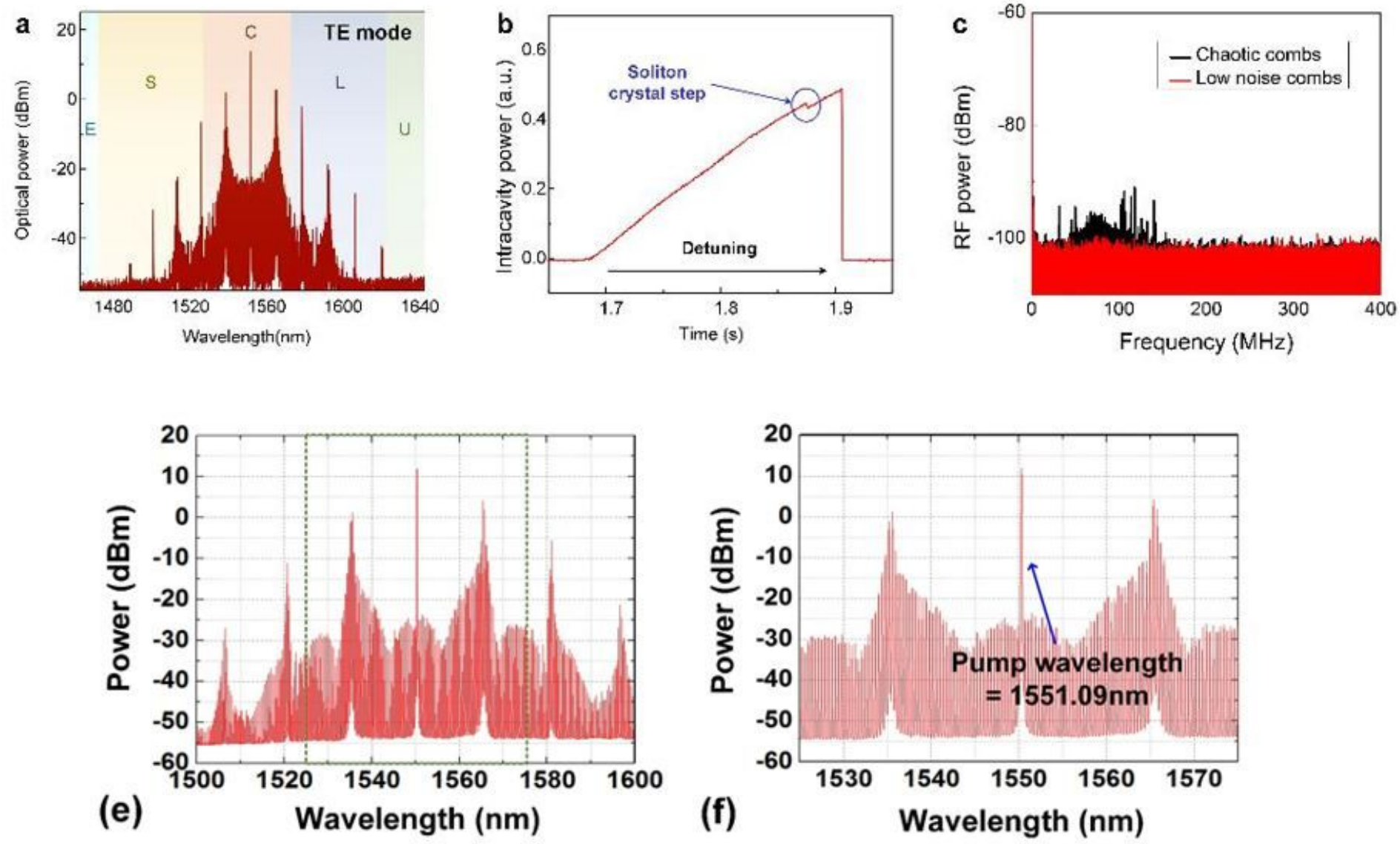

Figure 2

Optical spectra of various soliton crystal microcombs. the shaped micro-comb corresponding to the tap weights of centre frequency tunable Gaussian apodised sinc RF filters [27]. B) power output versus pump tuning, showing the very small power jump at the onset of soliton crystal combs. C) transition from high RF noise chaotic state to low noise state of the soliton crystal comb. 

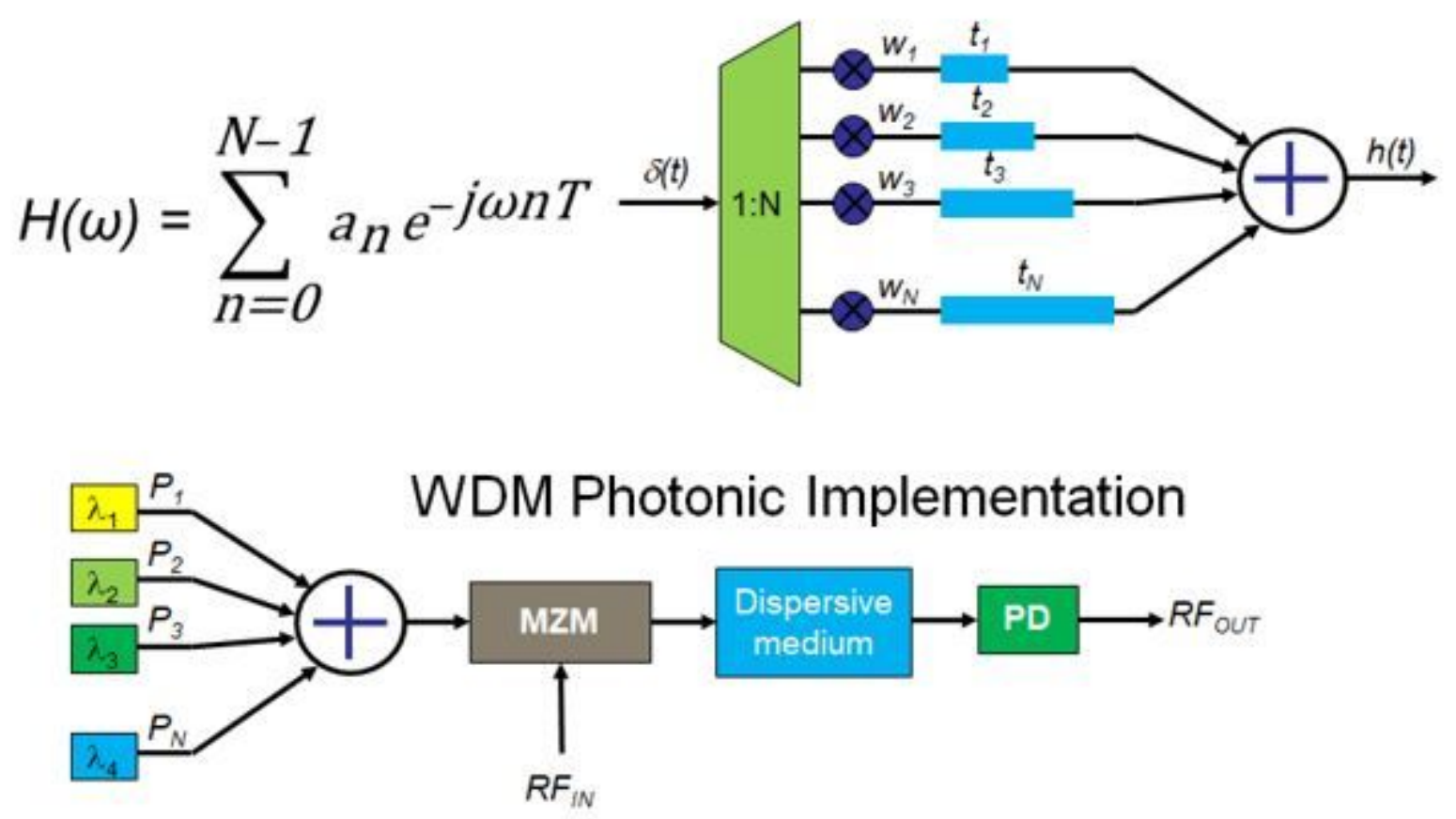

Figure 3

Theoretical schematic of the principle of transveral filters using wavelength multiplexing. MZM = mhach zhender modulators. 

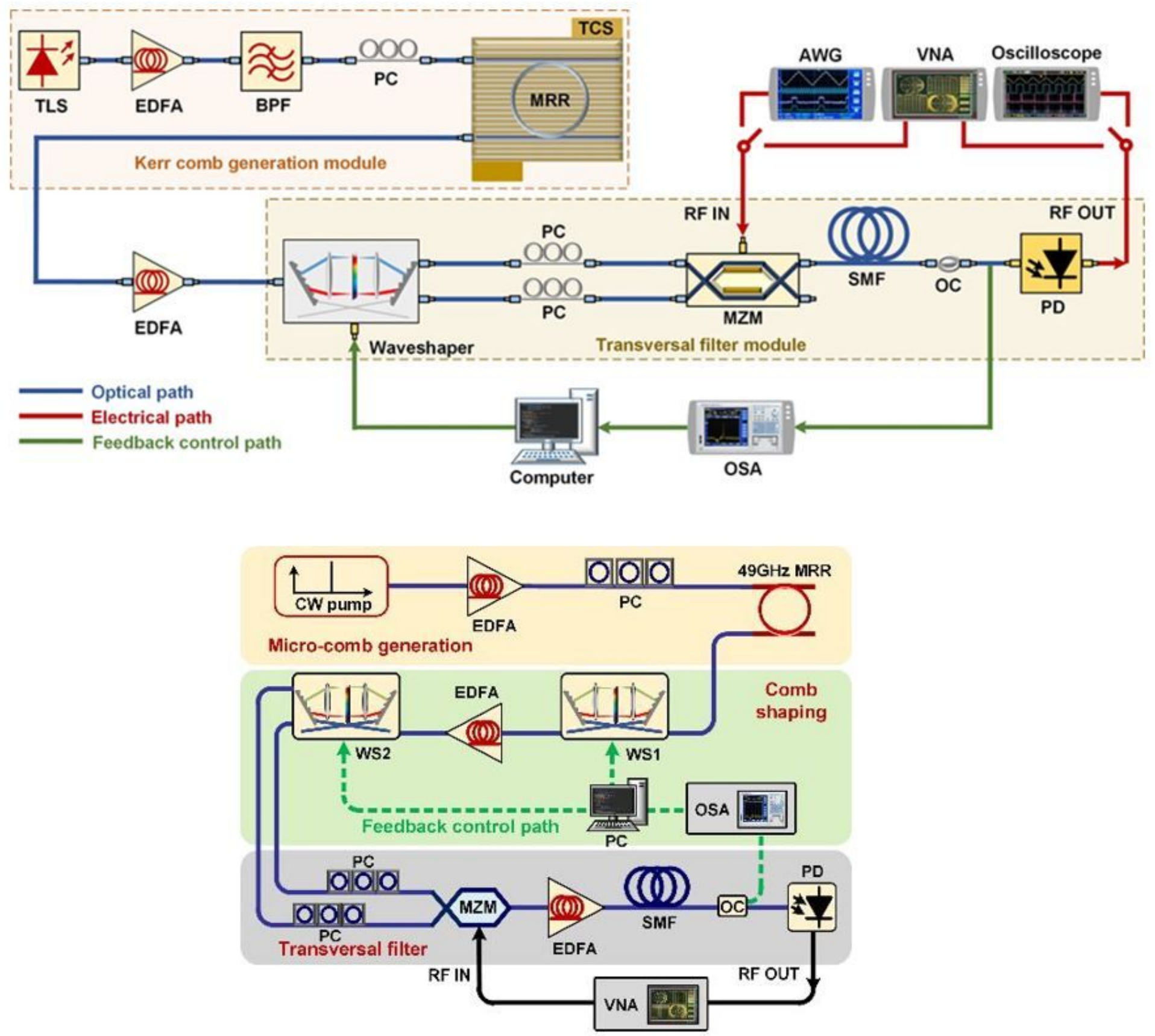

Figure 4

Experimental schematic for RF transversal filters based on $200 \mathrm{GHz}$ microcomb (top) and $49 \mathrm{GHz}$ microcomb (bottom). MZM: Mach-Zehnder modulator. EDFA: erbium-doped fiber amplifier. PC: polarization controller. MRR: micro-ring resonator. WS: Waveshaper. OC: optical coupler. OSA: optical spectrum analyzer. 


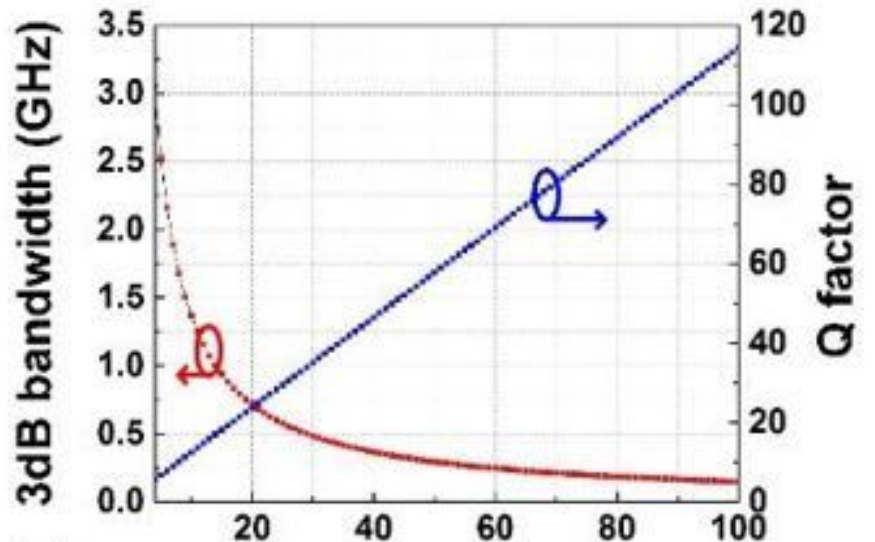

(a) Number of taps

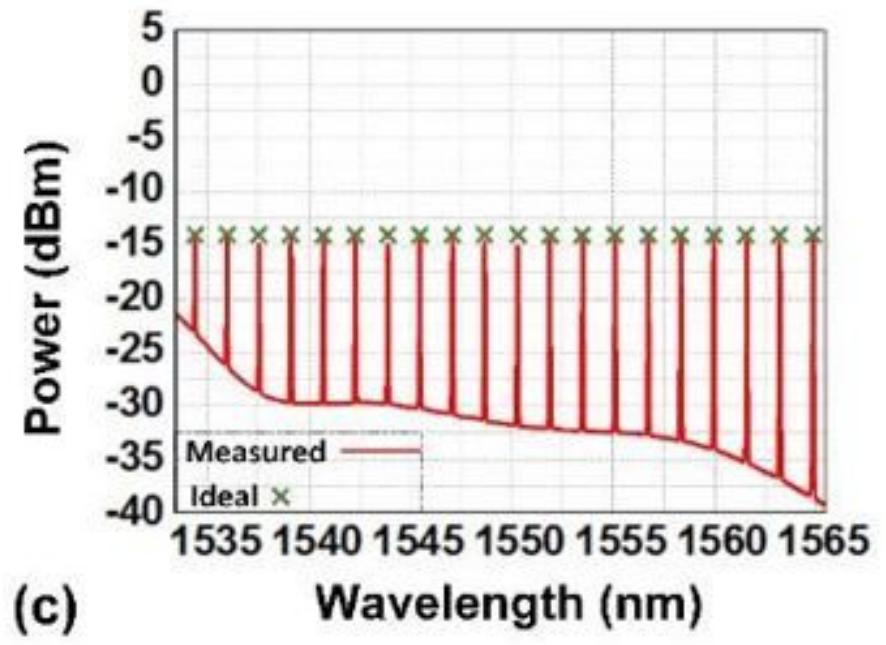

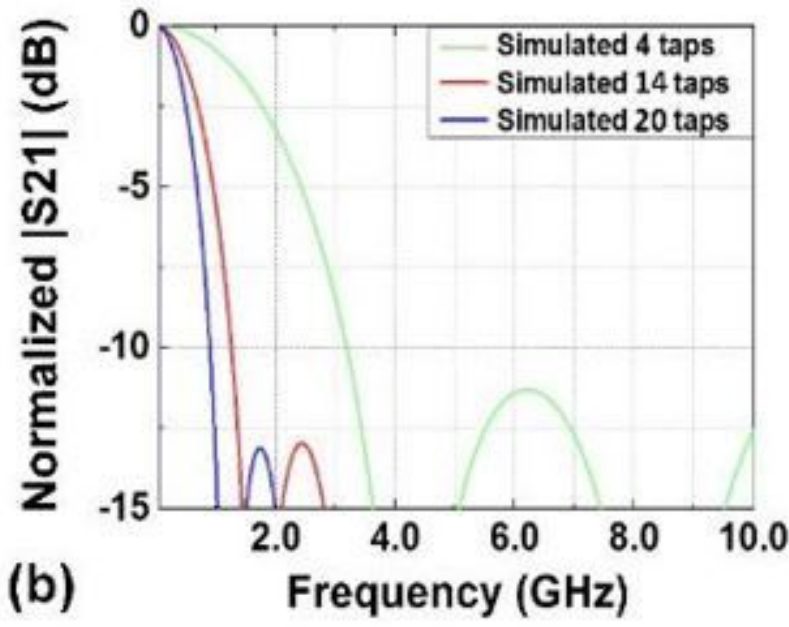

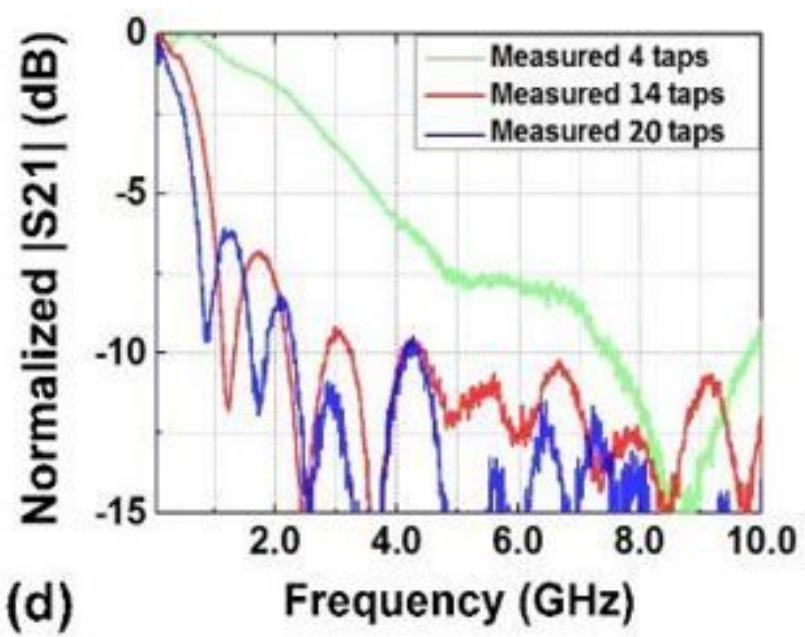

Figure 5

Fixed RF lowpass filters with uniform tap weights. 

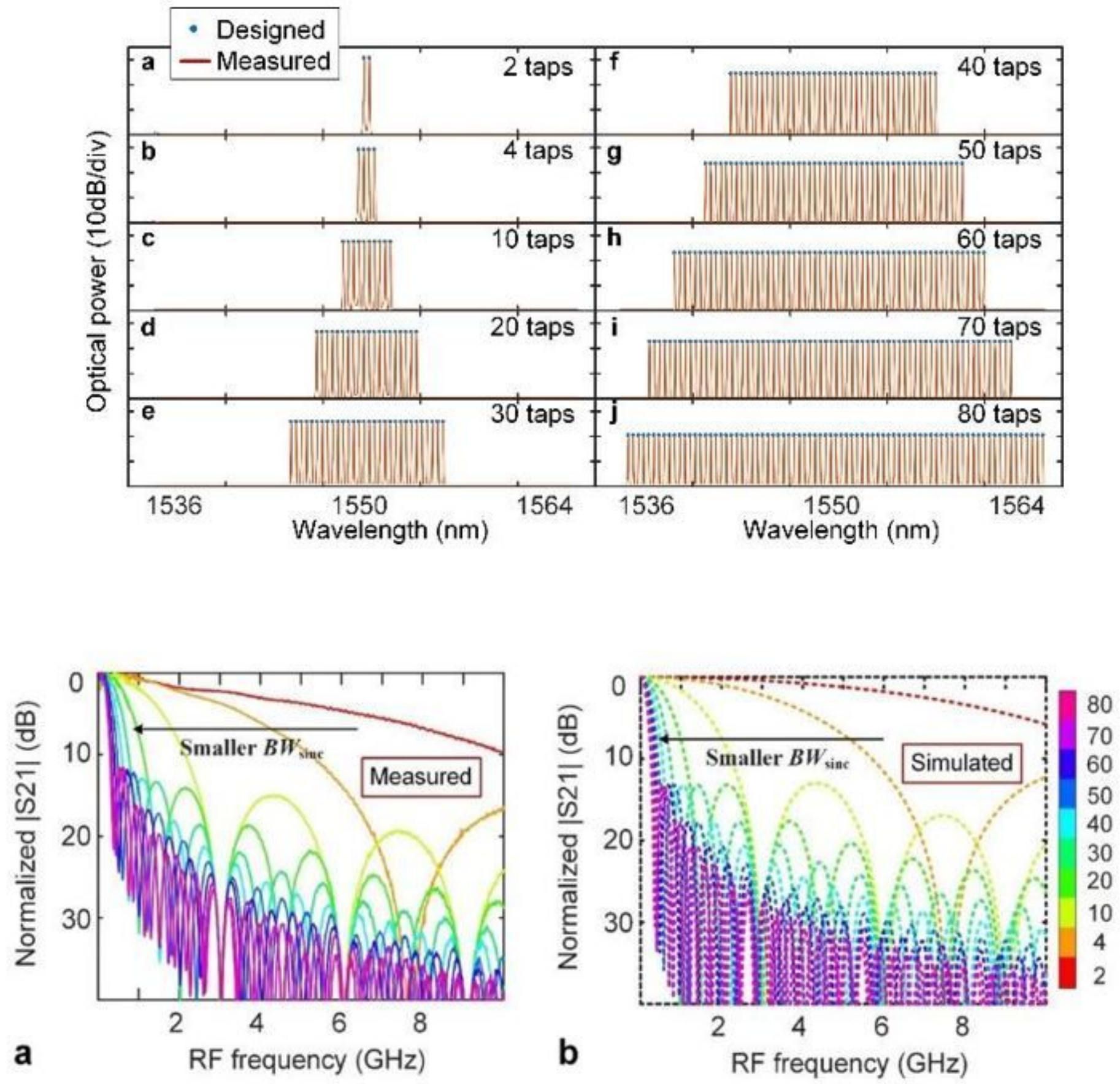

Figure 6

Fixed RF lowpass filters using the $49 \mathrm{GHz}$ FSR microcomb with up to 80 uniform tap weights. 


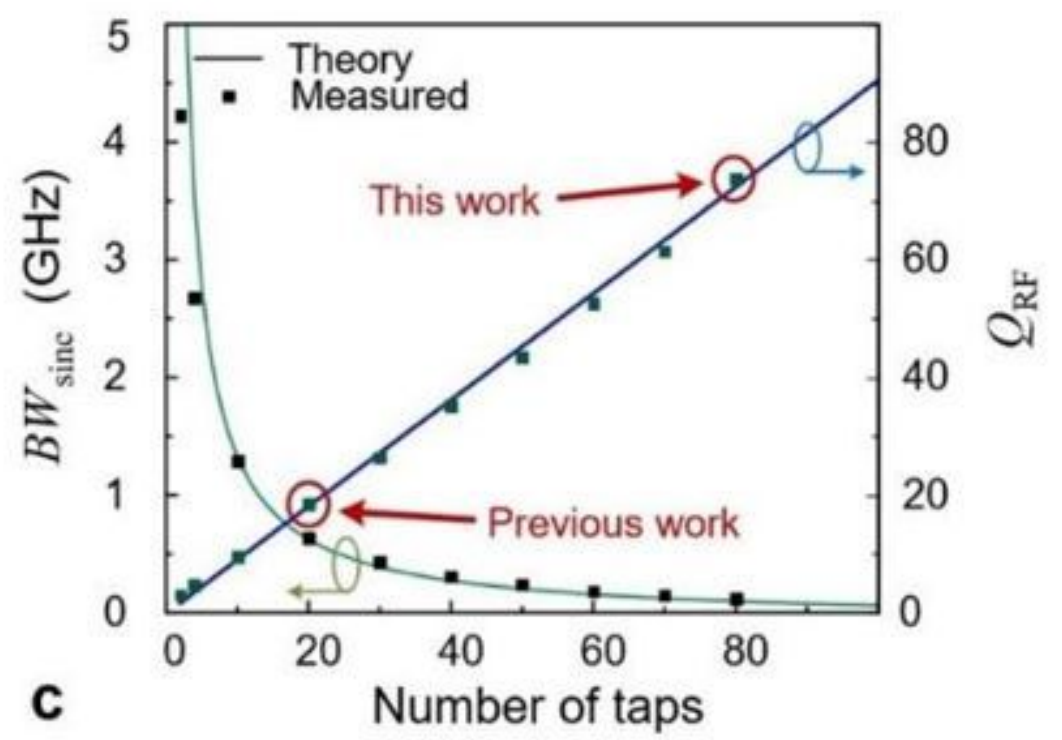

\section{Figure 7}

Calculated (solid curves) and measured (dots) RF filter bandwidth and Q factor versus the number of taps, showing the results for the $200 \mathrm{GHz}$ FSR device and the $49 \mathrm{GHz}$ FSR comb, with the latter having up to 80 taps. 

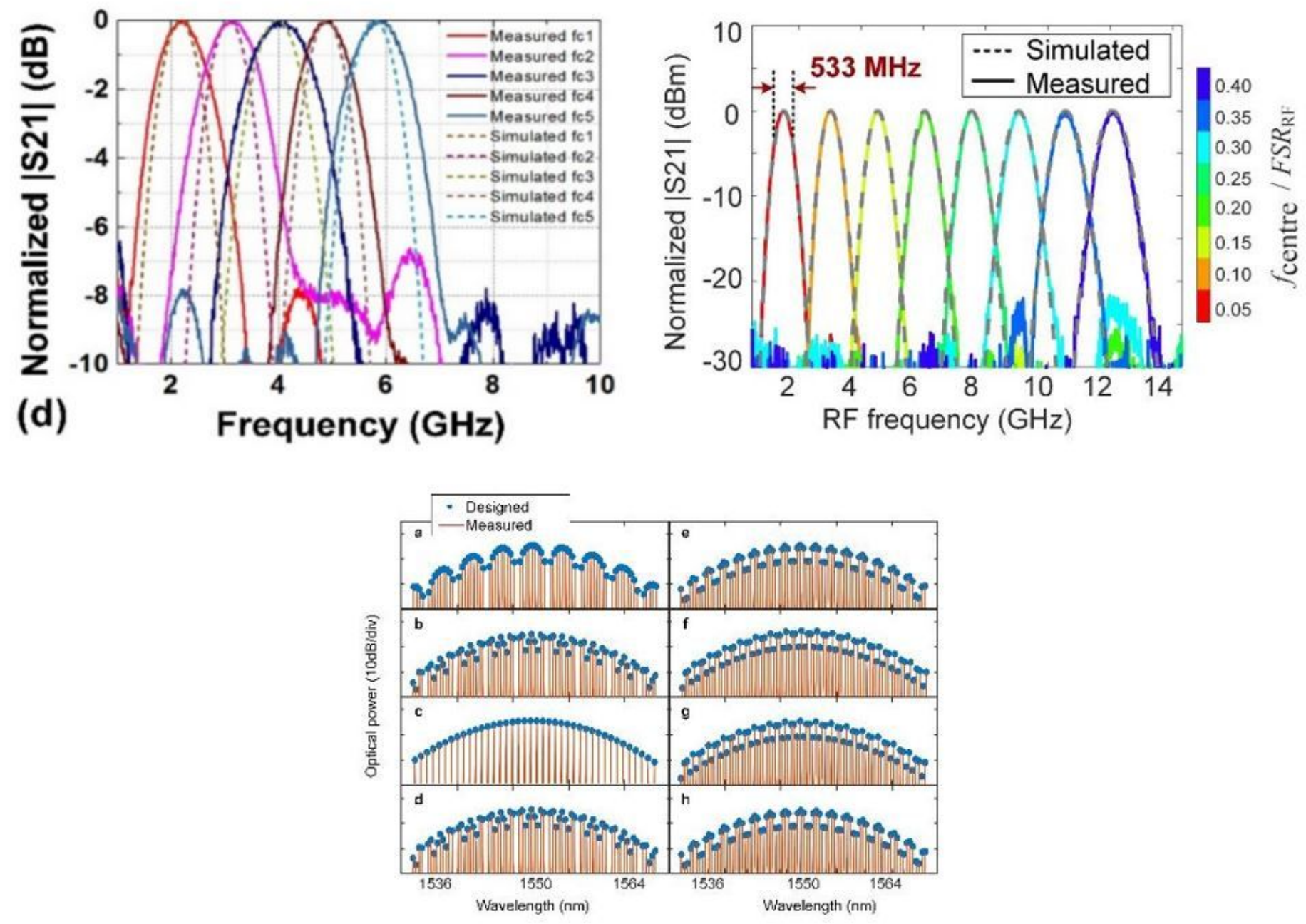

Figure 8

Optical spectra of the shaped micro-comb corresponding to the centre-frequency-tunable Gaussian apodized sinc filter for $200 \mathrm{GHz}$ FSR device (a) and $49 \mathrm{GHz}$ device. Bottom: designed tap weights for $49 \mathrm{GHz}$ FSR microcomb based tunable RF filter 

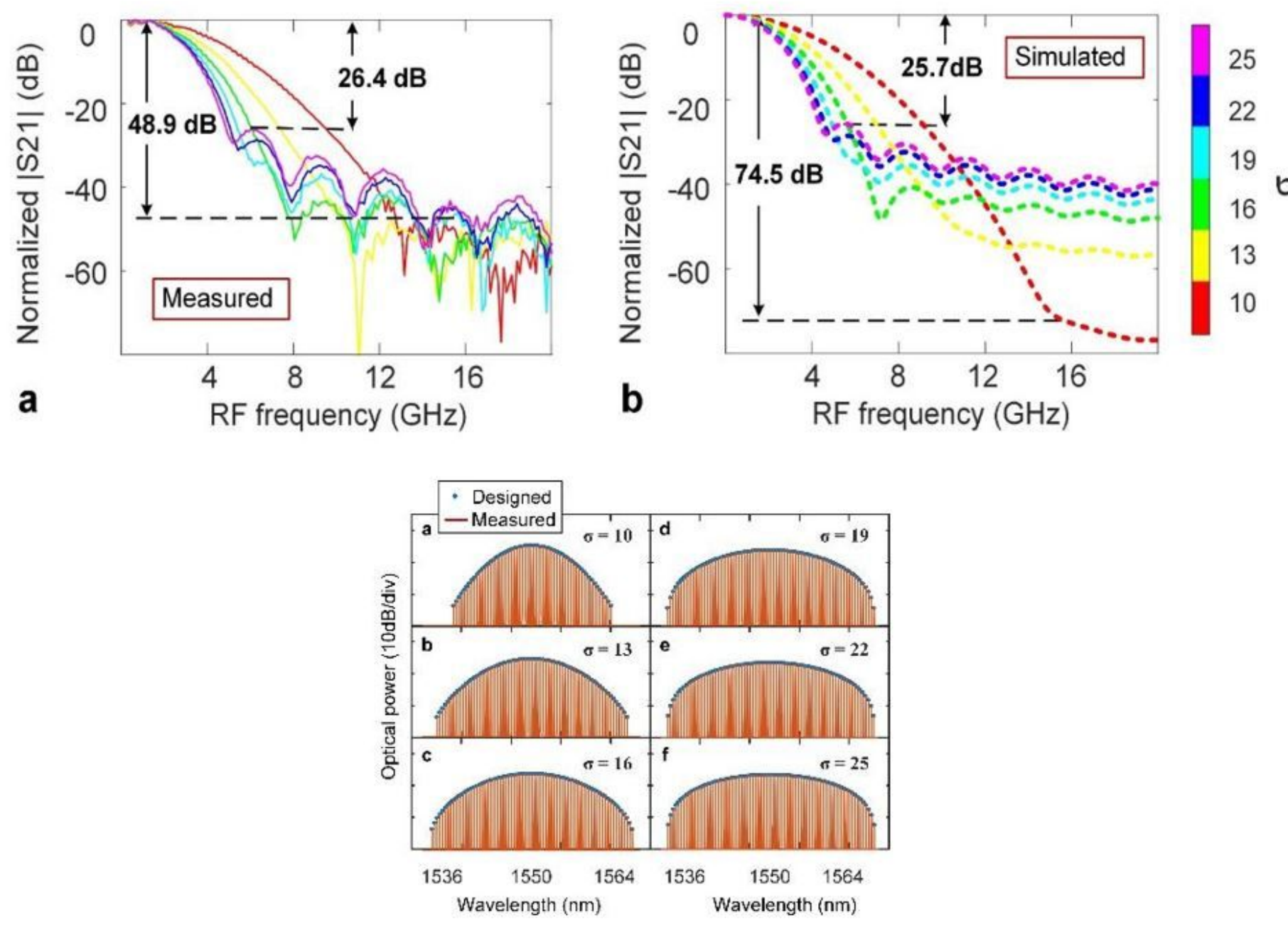

Figure 9

Optical spectra of the apodized RF filter (top) using the $49 \mathrm{GHz}$ FSR comb with up to 25 tap weights. Bottom: corresponding tap weights. 

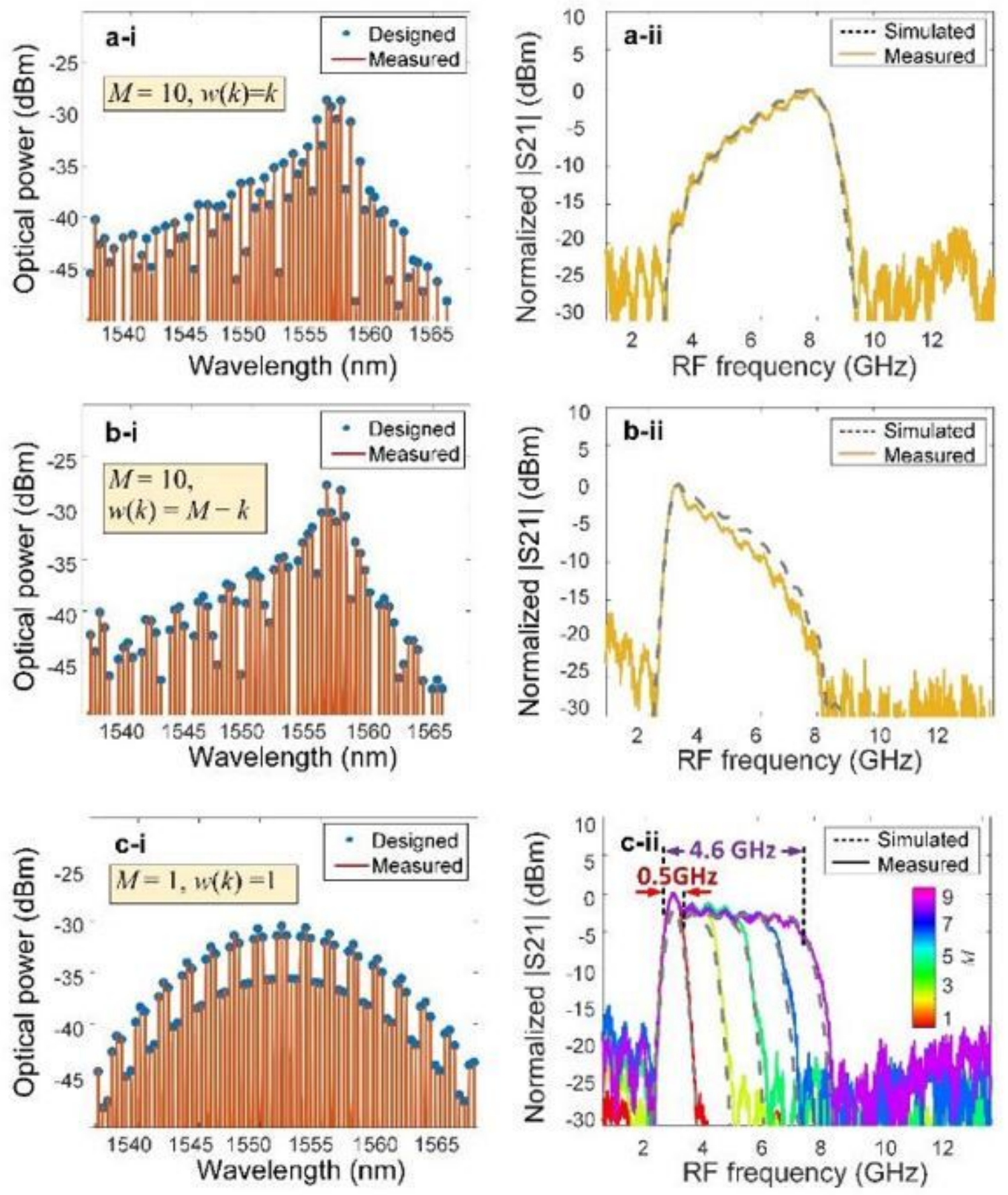

Figure 10

Optical spectra of the RF filters for non-uniform gain equalizing filters (a-d) showing the tap weights, as well as a variable bandpass filter (bottom), All are based on the 49GHz FSR comb with 80 tap weights.

\section{Supplementary Files}

This is a list of supplementary files associated with this preprint. Click to download. 
- formula.docx

Page 37/37 